\title{
Peaks are Preserved Under Run-Sorting
}

\author{
Per Alexandersson ${ }^{\dagger}$ and Olivia Nabawanda ${ }^{\ddagger}$ \\ ${ }^{\dagger}$ Department of Mathematics, Stockholm University, SE-106 91 Stockholm, Sweden \\ Email: per.w.alexandersson@gmail.com \\ $\ddagger$ Department of Mathematics, Makerere University, Kampala, Uganda \\ Email: onabawanda@must.ac.ug
}

Received: May 20, 2021, Accepted: June 15, 2021, Published: June 25, 2021 The authors: Released under the CC BY-ND license (International 4.0)

ABstRACT: We study a sorting procedure (run-sorting) on permutations, where runs are rearranged in lexicographic order. We describe a rather surprising bijection on permutations on length $n$, with the property that it sends the set of peak-values (also known as the pinnacle set) to the set of peak-values after run-sorting. We also prove that the expected number of descents in a permutation $\sigma \in S_{n}$ after run-sorting is equal to $(n-2) / 3$. Moreover, we provide a closed-form of the exponential generating function introduced by Nabawanda, Rakotondrajao, and Bamunoba in 2020, for the number of run-sorted permutations of $[n],(\mathcal{R S P}(n))$ having $k$ runs, which gives a new interpretation to the sequence http://oeis.org/A124324. We show that the descent generating polynomials, $A_{n}(t)$ for $\mathcal{R S P}(n)$ are real rooted, and satisfy an interlacing property similar to that satisfied by the Eulerian polynomials.

Finally, we study run-sorted binary words and compute the expected number of descents after run-sorting a binary word of length $n$.

Keywords: Flattened partition; Interlacing; Peak; Pinnacle set; Real-rootedness

2020 Mathematics Subject Classification: 05A05; 05A15

\section{Introduction and preliminaries}

Studying the distribution of different permutation statistics such as runs, descents, ascents, excedances, and peaks among others have a long history and have been an area of active research in enumerative combinatorics and mathematics at large, see e.g., $[9,14,15,18,19,29]$. Among the class of permutations where these statistics have been investigated are the so-called flattened partitions introduced by Callan, see [4]. Callan borrowed the word flatten from a function in Mathematica, where Flatten concatenates a list of lists into one single list.

Flattened partitions have received a lot of attention since then, see e.g., [17, 20-23]. Our main source of inspiration for this paper is the recent study on flattened partitions by Nabawanda, Rakotondrajao and Bamunoba [23].

Given a non-empty finite set $T$ of positive integers, a set partition $P$ of $T$ is a collection of disjoint non-empty subsets $B_{1}, B_{2}, \ldots, B_{k}$ of $T$ (which we simply call blocks) such that $B_{1} \cup B_{2} \cup \cdots \cup B_{k}=T$. To generate a flattened partition from $P$, the elements in each of the blocks of $P$ are first sorted increasingly, and then the blocks are arranged in lexicographical order. For instance, $\sigma=15672835$ is flattened as it arises from the set partition $1567|28| 35$, but $\sigma^{\prime}=4516732$ is not a flattened partition. Flattened partitions constitute a subset of $S_{n}$, the set of all permutations of $\{1,2, \ldots, n\}$. To be consistent, we shall instead refer to flattened partitions as run-sorted permutations, since we also consider run-sorted binary words in Section 5 . We let $\mathcal{R} \mathcal{S P}(n)$ denote the set of run-sorted permutations of length $n$.

For a fixed positive integer $n$, we set $[n]:=\{1,2, \ldots, n\}$. A permutation $\sigma \in S_{n}$ will be represented in the one-line notation, $\sigma(1) \sigma(2) \cdots \sigma(n)$. In particular, every permutation can be considered as a word of length $n$, with letters in $\mathbb{N}$.

We say that the word $w$ (of length $n$ ) has $k$ as a descent if $w(k)>w(k+1)$, where $k \in[n-1]$, where $n$ is the length of $w$. The descent set of $w$ is denoted by $\operatorname{DES}(w)$ and $\operatorname{des}(w)$ is the cardinality of $\operatorname{DES}(w)$. A closely related statistic is the set of descent bottoms;

$$
\operatorname{DB}(w):=\left\{w_{i+1}: i \in[n-1] \text { and } w_{i}>w_{i+1}\right\} .
$$


The set of left-to-right minima is defined as

$$
\operatorname{LRMin}(w):=\left\{w_{i}: w_{i}=\min \left\{w_{1}, w_{2}, \ldots, w_{i}\right\}\right\} .
$$

A word $w$ with exactly $r-1$ descents can be decomposed into $r$ (weakly) increasing subwords. Each of these subwords is a run of $w$. For $w=1526734$ (which is a permutation), we have the descents 2 and 5 , and the runs of $w$ are 15, 267 and 34. Given a word $w$, we let runsort $(w)$ be the word obtained by rearranging the runs of $w$ in lexicographic order. For example,

$$
\begin{aligned}
\operatorname{runsort}(297368514) & =142936857 \\
\operatorname{runsort}(1011011100011) & =0001101101111 .
\end{aligned}
$$

In particular, if $\sigma \in S_{n}$, then $\operatorname{runsort}(\sigma) \in \mathcal{R S P}(n)$.

In Section 2, Theorem 2.1, we refine a result by Nabawanda, Rakotondrajao and Bamunoba [23, Sec. 4], which describes a bijection between set partitions of $[n]$ and run-sorted permutation of size $n+1$. Our refinement allows us to compute the descent set of the resulting permutation from the set partition.

In Section 3, we describe the multivariate generating function for the descent set distribution of a run-sorted permutation $\sigma$. This solves the problem in [23], where an exponential generating function for the number of flattened partitions was described implicitly as a solution to a partial differential equation. We discover that our explicit exponential generating function produces a Sheffer sequence. We also show in Theorem 3.3 that the polynomials

$$
A_{n}(t):=\sum_{\sigma \in \mathcal{R S P}(n)} t^{\operatorname{des}(\sigma)}
$$

studied earlier in [23] are real-rooted. In fact, we show the stronger property that the roots of $A_{n-1}(t)$ interlace the roots of $A_{n}(t)$.

Definition 1.1 (Interlacing and alternating, see [28]). Let $f$ and $g$ be polynomials with positive leading coefficients and with real roots $\left\{f_{i}\right\}$ and $\left\{g_{i}\right\}$, respectively. We say that $f$ interlaces $g$ if $\operatorname{deg}(f)+1=\operatorname{deg}(g)=d$ and

$$
g_{1} \leq f_{1} \leq g_{2} \leq f_{2} \leq \cdots \leq f_{d-1} \leq g_{d}
$$

Moreover, we say that $f$ alternates left of $g$ if $\operatorname{deg}(f)=\operatorname{deg}(g)=d$ and

$$
f_{1} \leq g_{1} \leq f_{2} \leq \cdots \leq f_{d} \leq g_{d}
$$

We say that $f$ interleaves $g$ if either $f$ interlaces $g$ or $f$ alternates left of $g$. We write this as $f \ll g$. By convention, $0 \ll 0,0 \ll h$ and $h \ll 0$ whenever $h$ is a polynomial with a positive leading coefficient.

A peak of a permutation $\sigma \in S_{n}$, is an integer $i, 1<i<n$ such that $\sigma(i-1)<\sigma(i)>\sigma(i+1)$. If $i$ is a peak of $\sigma$, we say that $\sigma(i)$ is a peak-value of $\sigma$. For example, the permutation $\sigma=1374625 \in S_{7}$ has peak-values 7 and 6 with peaks 3 and 5 respectively. Let $\operatorname{PKV}(\sigma)$ denote the set of peak-values* of the permutation $\sigma$, and set $\operatorname{SPV}(\sigma):=\operatorname{PKV}(\operatorname{runsort}(\sigma))$.

The set of 132-peak-values is defined as

$$
\mathrm{PKV}^{*}(\pi):=\left\{\pi_{i}: 1<i<n \text { and } \pi_{i-1}<\pi_{i+1}<\pi_{i}\right\} .
$$

Note that $\operatorname{PKV}^{*}(\pi) \subseteq \operatorname{PKV}(\pi)$.

We shall also use the notation $\mathbf{x}_{T}:=x_{t_{1}} x_{t_{2}} \cdots x_{t_{m}}$ whenever $T=\left\{t_{1}, t_{2}, \ldots, t_{m}\right\}$ is a set of positive integers.

In Section 4, we prove the theorem below, which is the main result of this article.

Theorem 1.1. For any positive integer $n \geq 1$, then

$$
\sum_{\sigma \in S_{n}} \mathbf{x}_{\mathrm{PKV}(\sigma)}=\sum_{\tau \in S_{n}} \mathbf{x}_{\mathrm{SPV}(\tau)} .
$$

In fact, we prove a stronger version of this identity where an additional set-valued statistic is involved. This is achieved through a bijective proof, which exploits the recursive process of constructing a permutation in $S_{n}$ from a permutation in $S_{n-1}$ by inserting $n$ somewhere. We also prove that the expected number of descents ${ }^{\dagger}$ in a run-sorted permutation, $\sigma$ is given by

$$
\mathbb{E}[\operatorname{des}(\operatorname{runsort}(\sigma))]=\mathbb{E}[\operatorname{peaks}(\sigma)]=\frac{n-2}{3} .
$$

In Section 5, we prove that the number of permutations $\sigma \in S_{a+b}$ with major index $a$ and $b$ inversions, can be set in bijection with run-sorted binary words having $a$ 0's and $b 1$ 's. We are also able to compute the expected number of descents in runsort $(w)$, for $w$ being a uniformly chosen binary word of length $n$, see Section 5.4.

*This set is also known as the pinnacle set.

†We show that descents are always peaks in a run-sorted permutation. 


\section{Set partitions of $[n]$ and the descent set in a run-sorted permutation of $[n+1]$}

Let $\mathcal{S P}(n)$ be the set of set-partitions of $[n]$. Each element in $\mathcal{S P}(n)$ is expressed as $B_{1}\left|B_{2}\right| \cdots \mid B_{m}$, where the elements in each block are written in increasing order and the blocks arranged in lexicographic order.

Definition 2.1. We shall now recall a bijection sp-to-rsp : $\mathcal{S P}(n) \rightarrow \mathcal{R S P}(n+1)$ from [23, Sec, 4]. Given $P \in \mathcal{S P}(n)$, we first move the smallest element in each block to the end of the block. Then, all entries are increased by one, and we remove the vertical bars separating the blocks. This creates a word of length $n$. Finally, we prepend a 1 to the beginning of the word. The result is now an element in $\mathcal{R S P}(n+1)$.

Example 2.1. Consider the set partition $P=1258|3| 47 \mid 6$. Moving the smallest element in each block to the end of the block, then increasing the entries by 1 , and finally removing the vertical bars produces the word $w=36924857$, of length 8 . Inserting 1 at the beginning of $w$ gives $136924857 \in \mathcal{R S P}(9)$.

In [23], the following theorem was proved.

Theorem 2.1 (See [23, Thm. 19]). For any integer $n \geq 0$, if $P$ is a set-partition of $[n]$ and $\sigma=\operatorname{sp-to-rsp}(P)$, then the following assertions are equivalent:

(i) the number of blocks of size greater than 1 of the partition $P$ is $k-1$,

(ii) the number of runs in the flattened partition $\sigma$ is equal to $k$.

We shall refine Theorem 2.1 by describing how to obtain the set $\operatorname{DES}(\sigma)$ from the set partition corresponding to $\sigma$.

Proposition 2.1. Suppose $\sigma=\operatorname{sp-to-rsp~}(P)$ for some $P=B_{1}\left|B_{2}\right| \cdots \mid B_{m} \in \mathcal{S P}(n)$. For all $1 \leq i \leq m$, let $p_{i}$ denote the position of the last element in $B_{i}$. Then

$$
\operatorname{DES}(\sigma)=\left\{p_{i}: i \in[m] \text { where }\left|B_{i}\right| \geq 2\right\} .
$$

Proof. By Theorem 2.1, if there are $k$ blocks of size at least 2, we have that $|\operatorname{DES}(\sigma)|=k$, and $\sigma=\operatorname{sp}$-to-rsp $(P)$ has a total of $k+1$ runs. The lengths $l_{i}$, of the first $k$ runs of $\sigma$ are given by

$$
l_{i}=p_{i}-p_{i-1},
$$

with the convention $p_{0}:=0$. Moreover, $\sigma\left(p_{i}\right)$ is given by the relation

$$
\sigma\left(p_{i}\right)=p_{1}+n-i
$$

and we also have that $\sigma(1)=1$. The remaining elements of $\sigma$ are distributed from the set $\left\{2,3, \ldots, \sigma\left(p_{k}\right)-1\right\}$ in the same order, such that the lengths of the first $k$ runs are given by the values of the $l_{i}$. From these observations, we can draw the desired conclusion.

Remark 2.1. We should also note that the set of of peaks is the same as set of descents, for any element in $\mathcal{R S P}(n)$. Suppose $\sigma \in \mathcal{R S P}(n)$ and $k$ is a descent of $\sigma$, so that $\sigma(k)>\sigma(k+1)$. Now, since $\sigma$ is run-sorted, we must have that $\sigma(k-1)<\sigma(k)$, otherwise, $\sigma(k)$ is a run of length 1 in $\sigma$, violating Proposition 2.1. Hence, $k$ is also a peak of $\sigma$.

Example 2.2. Let us consider a set partition $P=18|27| 3|46| 5$ of $[8]$ having 3 blocks of size $\geq 2$ and let $\sigma=\operatorname{sp-to-rsp}(P)$. Then $\operatorname{DES}(\sigma)=\{2,4,7\}$. The lengths $l_{i}$ for $1 \leq i \leq 3$ of the first 3 runs of $\sigma$ are $l_{1}=2-0=2, l_{2}=4-2=2$, and $l_{3}=7-4=3$.

The last elements in each of the first 3 runs of $\sigma$, corresponding to each position in $\operatorname{DES}(\pi)$ are $\sigma\left(p_{1}\right)=$ $2+8-1=9, \sigma\left(p_{2}\right)=2+8-2=8$, and $\sigma\left(p_{3}\right)=2+8-3=7$.

Since $\sigma$ is run-sorted, $\sigma(1)=1$, and its remaining elements are chosen from the set $\{1,2, \ldots, 6\}$ and placed in this order. Consequently, $\sigma=192834756$.

\section{Run-sorted permutations and descent sets}

In this section, we describe multivariate generating functions for the descent set distribution of run-sorted permutations. The main result in this section is Theorem 3.2, where we provide the exponential generating function for flattened partitions of size $n$, where the number of descents is tracked. This resolves an open problem in [23], and we discover an interesting connection with Sheffer sequences.

We start with some recursions for tracking the descent set. 
Theorem 3.1. For all integers $n \geq 1$, let

$$
A_{n}(\mathbf{x}):=\sum_{\pi \in \mathcal{R} \mathcal{S P}(n)} \prod_{j \in \operatorname{DES}(\pi)} x_{n-j},
$$

so that we keep track of the descent set, by indexing from the end.

Then we have the recursive relations

$$
A_{n}(\mathbf{x})=1+\sum_{i=1}^{n-2}\left(\left(\begin{array}{c}
n-1 \\
i
\end{array}\right)-1\right) x_{i} A_{i}(\mathbf{x})
$$

and

$$
A_{n}(\mathbf{x})=A_{n-1}(\mathbf{x})+\sum_{i=1}^{n-2}\left(\begin{array}{c}
n-2 \\
i-1
\end{array}\right) x_{i} A_{i}(\mathbf{x}) .
$$

Before proving this, we shall look at a small example, because we use a somewhat non-standard convention.

Example 3.1. Let us compute the polynomial $A_{5}(\mathbf{x})$. Recall that the presence of $x_{i}$ indicates a descent at the $i^{\text {th }}$ position from the end. In Table 1, we compute the sum over all permutations in $\mathcal{R S P}(5)$, weighted by a monomial.

\begin{tabular}{ll}
$\pi \in \mathcal{R S P}(5)$ & $\prod_{j \in \operatorname{DES}(\pi)} x_{n-j}$ \\
\hline 12345 & 1 \\
$12354,12453,13452$ & $x_{1}$ \\
$12435,12534,13425,13524,14523$ & $x_{2}$ \\
$13245,14235,15234$ & $x_{3}$ \\
$13254,14253,15243$ & $x_{1} x_{3}$ \\
\hline
\end{tabular}

Table 1: The set $\mathcal{R} \mathcal{S P}(5)$ arranged according to the descent set. By summing the monomials in the second column, we see that $A_{5}(\mathbf{x})=1+3 x_{1}+5 x_{2}+3 x_{3}+3 x_{1} x_{3}$.

Proof of Theorem 3.1. We begin by proving the first relation. Note that there is only one run-sorted permutation with zero descents, i.e., the identity permutation. This corresponds to the 1 in the right-hand side of (3). Now consider a run-sorted permutation $\sigma \in \mathcal{R S P}(n)$ having at least one descent (and thus at least two runs). Let $i \in\{2,3, \ldots, n-1\}$ be the largest such descent (indexed from the end) meaning that the first run of $\sigma$ has length $n-i$. Removing the first run of $\sigma$, prepending a 1 , and then standardizing the result, produces a $\tau \in \mathcal{R S P}(i)$, where $\operatorname{des}(\sigma)=1+\operatorname{des}(\tau)$. Now, the elements greater than 1 in the first run of $\sigma$, is a subset of $\{2, \ldots, n\}$ of cardinality $n-i-1$. Moreover, any subset here is possible except $\{2,3, \ldots, n-i-1\}$, as in this case, there would not be a descent between the first and second run. It follows that given $\tau \in \mathcal{R S P}(i)$ and one of the $\left(\begin{array}{c}n-1 \\ n-i-1\end{array}\right)-1$ subsets of $\{2, \ldots, n\}$, we can uniquely recover $\sigma$, by simply inserting the elements in the chosen subset after the 1 in increasing order, and then standardizing the remaining entries so that a permutation is produced. This operation creates a new descent at position $i$ from the end, explaining the $x_{i}$ factor and we have proved (3).

For the identity in (4), we shall describe a bijection, that maps $\sigma \in \mathcal{R} \mathcal{S P}(n)$ to either some $\tau \in \mathcal{R} \mathcal{S P}(n-1)$ or to some $\tau \in \mathcal{R S P}(i)$ for some $i<n$, together with a subset of size $n-i-1$ of $\{3,4, \ldots, n\}$.

First case: 1 and 2 are in the same run of $\sigma$. We remove the 1 from $\sigma$ and decrease all entries by one. This produces $\tau \in \mathcal{R S P}(n-1)$, and $\operatorname{des}(\sigma)=\operatorname{des}(\tau)$.

Second case: 1 and 2 in different runs of $\sigma$. The second run of $\sigma$ must then necessarily start with 2 , and the first run of $\sigma$ contains 1 , and some subset of $\{3,4, \ldots, n\}$ of size $n-i-1$, for some $i \in[n-2]$. Note that $i$ is the number of elements in $\sigma$ not in the first run. We then let $\tau \in \mathcal{R S P}(i)$ be obtained from $\sigma$ by removing the first run, and then standardize the result. This preserves all descents (and their indexing from the end), except for the descent between the first and the second run of $\sigma$, which is located at position $i$ (from the end).

This procedure is invertible - given $\tau \in \mathcal{R S P}(i)$ and one of the $\left(\begin{array}{c}n-2 \\ n-i-1\end{array}\right)$ subsets of $\{3,4, \ldots, n\}$, there is a unique $\sigma \in \mathcal{R S P}(n)$ where the first run of $\sigma$ consists of 1 and the elements in the chosen subset. Hence, we have proved that $A_{n}(\mathbf{x})=A_{n-1}(\mathbf{x})+\sum_{i=1}^{n-2}\left(\begin{array}{c}n-2 \\ n-i-1\end{array}\right) x_{i} A_{i}(\mathbf{x})$, and by noting that $\left(\begin{array}{c}n-2 \\ n-i-1\end{array}\right)=\left(\begin{array}{c}n-2 \\ i-1\end{array}\right)$, we are done.

Example 3.2. Let us illustrate the proof of (3) and construct a run-sorted permutation of [6] having two descents from a run-sorted permutation $\tau$ of [3] having one descent at position 1 from the end. We have $\tau=132$. The allowed subsets of size two from the set $\{2,3,4,5,6\}$ are

$$
\{2,4\},\{2,5\},\{2,6\},\{3,4\},\{3,5\},\{3,6\},\{4,5\},\{4,6\},\{5,6\} .
$$

From the pair $(\{3,4\}, \tau)$, we then get $\pi=134265$, which has a descent at position 1 (from the end) and the second descent at position 3 (from the end). 
We now turn our attention to the univariate sequence $\left\{A_{n}(t)\right\}_{n \geq 0}$ of polynomials where $A_{n}(t)$ is obtained from $A_{n}(\mathbf{x})$ by letting $x_{i} \rightarrow t$. Equivalently,

$$
A_{n}(t):=\sum_{\pi \in \mathcal{R S P}(n)} t^{\operatorname{des}(\pi)}
$$

The first few such polynomials are given in Table 2 . From the definition, it is straightforward to see that $A_{n}(t)$ has degree $\lfloor n / 2\rfloor$.

\begin{tabular}{rr}
$n$ & $A_{n}(t)$ \\
\hline 1 & 1 \\
2 & 1 \\
3 & $t+1$ \\
4 & $4 t+1$ \\
5 & $3 t^{2}+11 t+1$ \\
6 & $25 t^{2}+26 t+1$ \\
7 & $15 t^{3}+130 t^{2}+57 t+1$ \\
8 & $210 t^{3}+546 t^{2}+120 t+1$ \\
9 & $105 t^{4}+1750 t^{3}+2037 t^{2}+247 t+1$ \\
\hline
\end{tabular}

Table 2: The polynomials $A_{n}(t)$.

Now let $f_{n, k}$ be the number of run-sorted permutations of $[n]$ having $k$ runs, see http://oeis.org/A124324 in the OEIS. Note that

$$
t A_{n}(t)=\sum_{\pi \in \mathcal{R S P}(n)} t^{\operatorname{des}(\pi)+1}=\sum_{k \geq 1} t^{k} f_{n, k}
$$

In [23] the authors proved that for all integers $n$ and $k$ such that $1 \leq k \leq n$, we have

$$
f_{n+2, k}=f_{n+1, k}+\sum_{i=1}^{n}\left(\begin{array}{c}
n \\
i
\end{array}\right) f_{n+1-i, k-1} .
$$

Using this recurrence relation, the exponential generating function, $F(t, u):=\sum_{n, k \geq 0} \frac{f_{n, k}}{n !} u^{n} t^{k}$ for these numbers was found to satisfy

$$
\frac{\partial F(t, u)}{\partial u}=t \exp (t(\exp (u)-1)+u(1-t)) \operatorname{with} \frac{\partial F(t, 0)}{\partial u}=t .
$$

We observe that $F(t, u)$ is not in its closed-form, and the authors of this article left this as an open problem to find a closed-form solution.

We now use the recurrence relation in (3), to instead find the generating function for the polynomials $A_{n+1}(t)$, where we have shifted the index by one. This modification allows us to derive a closed-form for the corresponding exponential generating function.

Theorem 3.2. Let $G(t, u)$ be the exponential generating function

$$
G(t, u)=\sum_{n \geq 0} A_{n+1}(t) \frac{u^{n}}{n !}
$$

Then

$$
G(t, u)=\exp \left[u+t\left(e^{u}-u-1\right)\right] .
$$

Proof. The recursion in (4) gives that

$$
\sum_{n \geq 0} A_{n+1}(t) \frac{u^{n}}{n !}=\sum_{n \geq 0} A_{n}(t) \frac{u^{n}}{n !}+t \sum_{n \geq 0} \sum_{j=1}^{n-1} A_{j}(t) \frac{(n-1) !}{(j-1) !(n-j) !} \frac{u^{n}}{n !} .
$$

Rewriting now gives

$$
G(t, u)=\sum_{n \geq 0} A_{n}(t) \frac{u^{n}}{n !}+t \sum_{j \geq 1} \sum_{n \geq j+1} A_{j}(t) \frac{(n-1) !}{(j-1) !(n-j) !} \frac{u^{n}}{n !} .
$$

We differentiate both sides with respect to $u$, and get

$$
\frac{\partial}{\partial u} G(t, u)=\sum_{n \geq 1} A_{n}(t) \frac{u^{n-1}}{(n-1) !}+t \sum_{j \geq 1} \sum_{n \geq j+1} A_{j}(t) \frac{(n-1) !}{(j-1) !(n-j) !} \frac{u^{n-1}}{(n-1) !} .
$$


This leads to

$$
\frac{\partial}{\partial u} G(t, u)-G(t, u)=t \sum_{j \geq 1} \frac{A_{j}(t) u^{j-1}}{(j-1) !} \sum_{n \geq j+1} \frac{u^{n-j}}{(n-j) !} .
$$

The inner sum is simply $e^{u}-1$, so

$$
\frac{\partial}{\partial u} G(t, u)-G(t, u)=t\left(e^{u}-1\right) \sum_{j \geq 1} \frac{A_{j}(t) u^{j-1}}{(j-1) !}=t\left(e^{u}-1\right) G(t, u) .
$$

Hence, we arrive at the differential equation $\frac{\partial G}{\partial u}=\left(1+t\left(e^{u}-1\right)\right) G$. It is now straightforward to verify that the function in (9) is the solution to this differential equation we seek.

As a final observation, a polynomial sequence $\left\{S_{n}(t)\right\}_{n \geq 0}$ is said to be Sheffer if and only if its generating function has the form

$$
\sum_{n=0}^{\infty} \frac{S_{n}(t)}{n !} u^{n}=P(u) e^{t \cdot Q(u)}
$$

where $P(u)=P_{0}+P_{1} u+P_{2} u^{2}+\cdots$ and $Q(u)=Q_{1}(u)+Q_{2} u^{2}+\cdots$ with $P_{0}, Q_{1} \neq 0$, see [25, 27]. From the generating function $G(t, u)$ in $(9)$, it follows that the sequence $\left\{A_{n+1}(t) / n !\right\}_{n \geq 0}$ is indeed a Sheffer sequence with $P(u)=e^{u}$ and $Q(u)=e^{u}-u-1$.

Question 3.1. Can we use properties of Sheffer sequences to deduce additional information about the polynomials $\left\{A_{n}(t)\right\}_{n \geq 1}$ ? Sheffer sequences are closely related to umbral calculus and shift operators. Does this carry over to the combinatorial side here in a meaningful way?

\subsection{Realrootedness and interlacing roots}

It is well-known that the Eulerian polynomials, $E_{n}(t)$, defined as

$$
E_{n}(t):=\sum_{\sigma \in S_{n}} t^{\operatorname{des}(\sigma)}
$$

are all real-rooted. In this subsection, we shall prove that our polynomials $A_{n}(t)$ are also real-rooted. It will be convenient to work with $R_{n}(t):=t A_{n}(t)$ as in (6), and we recall the definition of $f_{n, k}$ from there.

In [23], it was proved that the numbers $f_{n, k}$ satisfy the recurrence relation

$$
f_{n, k}=k f_{n-1, k}+(n-2) f_{n-2, k-1}, \text { whenever } 1 \leq k<n .
$$

Lemma 3.1. We have that the $R_{n}(t)$ satisfy the recurrence

$$
R_{n}(t)=t R_{n-1}^{\prime}(t)+t(n-2) R_{n-2}(t)
$$

with initial conditions $R_{1}(t)=R_{2}(t)=t$.

Proof. By (11), we have that

$$
\begin{aligned}
R_{n}(t) & =\sum_{k} t^{k}\left(k f_{n-1, k}+(n-2) f_{n-2, k-1}\right)=\sum_{k} t^{k} k f_{n-1, k}+\sum_{k}(n-2) t^{k} f_{n-2, k-1} \\
& =t \sum_{k} k t^{k-1} f_{n-1, k}+t(n-2) \sum_{k-1} t^{k-1} f_{n-2, k-1} .
\end{aligned}
$$

This is now recognized as (12).

Our goal is now to use (12) to show that all $R_{n}(t)$ are real-rooted. We shall in fact prove a much stronger property, namely that $R_{n-1}(t)$ either interlaces or alternates to the left of $R_{n}(t)$ for all $n \geq 1$.

The following lemma is our main tool for proving this statement - these properties are easy to prove by using the mean value theorem for example.

Lemma 3.2 (See D.Wagner, [28, Sec. 3]). Let $f, g, h \in \mathbb{R}[t]$ be real-rooted polynomials with only real, nonpositive roots and positive leading coefficients. Then

(i) if $f \ll h$ and $g \ll h$ then $f+g \ll h$.

(ii) if $h \ll f$ and $h \ll g$ then $h \ll f+g$.

(iii) $g \ll f$ if and only if $f \ll t g$. 
We are now ready to prove the main result in this section.

Theorem 3.3. The polynomials

$$
R_{n}(t)=\sum_{\pi \in \mathcal{R S P}(n)} t^{\operatorname{des}(\pi)+1}
$$

satisfy $R_{n-1} \ll R_{n}$ for all $n \geq 1$. In particular, they are all real-rooted.

Proof. We first note that the statement is true for $n=1$, since $R_{0}(t)=0$ and $R_{1}(t)=t$. We shall proceed by induction over $n$, so fix $n \geq 2$ and assume that we have $R_{n-2} \ll R_{n-1}$.

It suffices to prove that $R_{n-1} \ll R_{n}$. By Rolle's theorem, we know that $R_{n-1}^{\prime}$ interlaces $R_{n-1}$ i.e., $R_{n-1}^{\prime} \ll$ $R_{n}$. Together with the induction hypothesis and (iii) in Lemma 3.2, we have

$$
t R_{n-1}^{\prime} \ll R_{n-1} \text { and } t(n-2) R_{n-2} \ll R_{n-1} \text {. }
$$

Now, by (ii) in Lemma 3.2, we have that

$$
R_{n-1} \ll t R_{n-1}^{\prime}+t(n-2) R_{n-2},
$$

and by using (12), we can now conclude that $R_{n-1} \ll R_{n}$.

The preceding result also implies that $A_{n-1} \ll A_{n}$ for all $n>1$, as $A_{n}(t)=R_{n}(t) / t$. In particular, all the $A_{n}$ are real-rooted.

\subsection{Conjectures on stability}

Let $\mathcal{H} \subset \mathbb{C}$ denote the upper half-plane $\{z \in \mathbb{C}: \operatorname{im}(z)>0\}$. A multivariate polynomial $P \in \mathbb{C}\left[z_{1}, \ldots, z_{n}\right]$ is called stable if it does not vanish on $\mathcal{H}^{n}$. That is, $P$ is stable if

$$
\mathbf{z}^{*} \in \mathcal{H}^{n} \Longrightarrow P(\mathbf{z}) \neq 0 \text {. }
$$

One can easily show that $P$ is stable if and only if $P(\alpha+\lambda t)=0$ has only real zeros for all $\alpha \in \mathbb{R}^{n}, \lambda \in \mathbb{R}_{+}^{n}$. Note that a univariate polynomial with real coefficients is stable if and only if all roots are real.

The following theorem is attributed to P. Brändén, see [13, Thm. 2.5], and [1].

Theorem 3.4. Let $\tilde{E}_{n}(\mathbf{x})$ be defined as

$$
\tilde{E}_{n}(\mathbf{x}):=\sum_{\pi \in S_{n}} \prod_{\pi_{j}>\pi_{j+1}} x_{\pi_{j}} .
$$

Then $\tilde{E}_{n}(\mathbf{x})$ is stable.

Note that setting $x_{i} \rightarrow t$, we recover the classical Eulerian polynomials in (10), so Bränden's result implies that the Eulerian polynomials are real-rooted.

The following notion was introduced in [16] and is a strictly weaker notion than stability.

Definition 3.1 (Same-phase stability). A polynomial $p\left(x_{1}, \ldots, x_{n}\right) \in \mathbb{R}\left[x_{1}, \ldots, x_{n}\right]$ is said to be same-phase stable if for every $\lambda \in \mathbb{R}_{+}^{n}$, we have that the univariate polynomial $p\left(\lambda_{1} t, \lambda_{2} t, \ldots, \lambda_{n} t\right) \in \mathbb{R}[t]$ is real-rooted.

Definition 3.2 (Interlacing sequences of polynomials). A sequence $\left\{f_{1}, f_{2}, \ldots, f_{n}\right\}$ of polynomials with positive leading coefficients is said to be an interlacing sequence ${ }^{\ddagger}$ if $f_{i} \ll f_{j}$ for all $1 \leq i<j \leq n$. We let $\mathcal{F}_{n}^{+}$denote the set of all such interlacing sequences.

Let $E_{n}(\mathbf{x})$ be the multivariate Eulerian polynomial

$$
E_{n}(\mathbf{x}):=\sum_{\pi \in S_{n}} \mathbf{x}_{\operatorname{DES}(\pi)} .
$$

In contrast with the $\tilde{E}_{n}(\mathbf{x})$ we mentioned earlier, the $E_{n}(\mathbf{x})$ are not stable. For example

$$
\begin{aligned}
E_{5}(\mathbf{x}) & =6 x_{2} x_{1}+4 x_{2} x_{3} x_{1}+16 x_{3} x_{1}+9 x_{2} x_{4} x_{1}+x_{2} x_{3} x_{4} x_{1}+9 x_{3} x_{4} x_{1}+11 x_{4} x_{1} \\
& +4 x_{1}+9 x_{2}+11 x_{2} x_{3}+9 x_{3}+16 x_{2} x_{4}+4 x_{2} x_{3} x_{4}+6 x_{3} x_{4}+4 x_{4}+1
\end{aligned}
$$

vanishes at

$$
x_{1}=-\frac{39}{16}+\frac{7 i}{512}, x_{2}=-16+i, x_{3}=i, x_{4}=\frac{-6523999+73341 i}{5671874} .
$$

However, we shall show that the $E_{n}(\mathbf{x})$ are same-phase stable, and satisfy a type of interlacing.

\footnotetext{
${ }^{\ddagger}$ Yes, we also think this is rather unfortunate terminology, but here we follow [3].
} 
Theorem 3.5. Let $\lambda_{1}, \lambda_{2}, \ldots$ be a fixed sequence of positive real numbers. Then for all $n \geq 1$,

$$
E_{n-1}\left(\lambda_{1} t, \lambda_{2} t, \ldots, \lambda_{n} t\right) \ll E_{n}\left(\lambda_{1} t, \lambda_{2} t, \ldots, \lambda_{n} t\right) .
$$

Proof. Let us first refine the polynomial $E_{n}(\mathbf{x})$ by introducing

$$
E_{n}^{i}(\mathbf{x}):=\sum_{\substack{\pi \in S_{n} \\ \pi(n)=i}} \mathbf{x}_{\operatorname{DES}(\pi)} .
$$

We have that $E_{n-1}(\mathbf{x})=E_{n}^{n}(\mathbf{x})$, since removing the last entry (which is $n$ ) in a permutation counted by $E_{n}^{n}(\mathbf{x})$, gives a bijection with elements counted by $E_{n-1}(\mathbf{x})$. In order to make expressions more compact later, we set

$$
v_{n}^{i}(t):=E_{n}^{i}\left(\lambda_{1} t, \lambda_{2} t, \ldots, \lambda_{n} t\right), \quad v_{n}(t):=E_{n}\left(\lambda_{1} t, \lambda_{2} t, \ldots, \lambda_{n} t\right) .
$$

We shall now prove that $\mathcal{V}_{n}:=\left\{v_{n}^{i}(t)\right\}_{i=1}^{n}$ is an interlacing sequence. We follow the same approach as in $[3$, Ex. 7.8.8], and note that by conditioning on $\pi(n-1)=k$, we have

$$
v_{n+1}^{i}(t)=\sum_{k \geq i} \lambda_{n} t \cdot v_{n}^{k}(t)+\sum_{k<i} v_{n}^{k}(t)
$$

Note that the weak inequality in the second sum is due to standardization. For example, if $i=2$, and $\pi=4356172$, then $\pi$ is obtained from the standardization of $\pi$ with the last element dropped, e.g., 324516 .

We now rephrase the recursion in (13) as

$$
\left[\begin{array}{c}
v_{n+1}^{n+1} \\
v_{n+1}^{n} \\
v_{n+1}^{n-1} \\
\vdots \\
v_{n+1}^{3} \\
v_{n+1}^{2} \\
v_{n+1}^{1}
\end{array}\right]=\left[\begin{array}{cccccc}
1 & 1 & 1 & \ldots & 1 & 1 \\
\lambda_{n} t & 1 & 1 & \ldots & 1 & 1 \\
\lambda_{n} t & \lambda_{n} t & 1 & \ldots & 1 & 1 \\
\lambda_{n} t & \lambda_{n} t & \lambda_{n} t & \ldots & 1 & 1 \\
\vdots & \vdots & & \ddots & \ddots & \vdots \\
\lambda_{n} t & \lambda_{n} t & \ldots & \lambda_{n} t & \lambda_{n} t & 1 \\
\lambda_{n} t & \lambda_{n} t & \ldots & \lambda_{n} t & \lambda_{n} t & \lambda_{n} t
\end{array}\right]\left[\begin{array}{c}
v_{n}^{n} \\
v_{n}^{n-1} \\
\vdots \\
v_{n}^{3} \\
v_{n}^{2} \\
v_{n}^{1}
\end{array}\right]
$$

where we denote the big matrix by $G_{n} \in \mathbb{R}^{(n+1) \times n}$. We now use [3, Thm. 7.8.5], which allows us to easily verify that $G_{n}$ maps $\mathcal{F}_{n}^{+}$to $\mathcal{F}_{n+1}^{+}$, so (by induction) it follows that $\mathcal{V}_{n}$ is an interlacing sequence for all $n$ since the base case $\mathcal{V}_{1}=(1)$ is trivially an interlacing sequence.

We now have that

$$
v_{n-1}(t)=v_{n}^{n}(t) \text { and } v_{n}(t)=v_{n}^{1}(t)+\cdots+v_{n}^{n}(t) .
$$

Since $v_{n-1}(t)$ interleaves all polynomials in $\left\{v_{n}^{i}(t)\right\}_{i=1}^{n}$, it must also interleave the sum, so $v_{n-1} \ll v_{n}(t)$, and we are done.

Conjecture 3.1. Let $A_{n}(\mathbf{x})$ be as in (3). Then $A_{n}(\mathbf{x})$ is same-phase stable, and for all $n \geq 1$, we have that

$$
A_{n-1}\left(\lambda_{1} t, \lambda_{2} t, \ldots, \lambda_{n-1} t\right) \ll A_{n}\left(\lambda_{1} t, \lambda_{2} t, \ldots, \lambda_{n} t\right),
$$

whenever $\lambda_{1}, \lambda_{2}, \ldots$ is a fixed sequence of positive real numbers.

Again, same-phase stability is the best we can hope for. We have that $A_{5}(\mathbf{x})=1+3 x_{1}+5 x_{2}+3 x_{3}+3 x_{1} x_{3}$, but $A_{5}(-3+i, 11 / 5+3 i / 5, i, i)=0$, so $A_{5}(\mathbf{x})$ has a zero in $\mathcal{H}^{4}$, so $A_{5}(\mathbf{x})$ is not stable.

As in the proof of Theorem 3.5 , a possible approach is to refine $A_{n}(\mathbf{x})$ by introducing

$$
A_{n}^{i}(\mathbf{x}):=\sum_{\substack{\pi \in \mathcal{R} \mathcal{S P}(n) \\ \pi(2)=i}} \prod_{j \in \operatorname{DES}(\pi)} \mathbf{x}_{n-j} .
$$

Now let $\lambda_{1}, \lambda_{2}, \ldots$ be positive real numbers and let $n$ be fixed. Set

$$
a_{n}^{i}(t):=A_{n}^{i}\left(\lambda_{1} t, \lambda_{2} t, \ldots, \lambda_{n} t\right) .
$$

Computer experiments now suggest that $\left\{a_{n}^{i}(t)\right\}_{i=1}^{n}$ is an interlacing sequence.

Another approach is to refine based on the last entry of $\pi$ as we did in Theorem 3.5 produces the polynomials

$$
\sum_{\substack{\pi \in \mathcal{R S P}(n) \\ \pi(n)=i}} \prod_{j \in \operatorname{DES}(\pi)} \lambda_{n-j} t, \quad 2 \leq i \leq n,
$$

which also seem to be real-rooted. However, they do not form an interlacing sequence. 


\section{The peak-value distribution is preserved under run-sort}

We study the distribution of descents after run-sorting a permutation. In Section 4.1, we first give some historical background and results concerning peaks in permutations. We then prove the main technical results in Section 4.2, which amount to a type of bijective argument. In Section 4.3, we use the previous arguments to prove the main result of this article, and in the final subsection, we study some consequences of our result.

\subsection{Peaks in permutations}

We let $\hat{b}_{n, k}$ be the number of permutations in $S_{n}$ with exactly $k$ peaks. There is a nice recursion for the numbers $\hat{b}_{n, k}$ given first in [15, p.24], and has been discovered numerous times since then. We have that

$$
\hat{b}_{n, k}= \begin{cases}1 & \text { if } n=1, k=0 \\ (2 k+2) \hat{b}_{n-1, k}+(n-2(k-1)-2) \hat{b}_{n-1, k-1} & \text { if } 0 \leq k<\frac{n}{2} \\ 0 & \text { if } 2 k \geq n \text { or } k<0\end{cases}
$$

These numbers can be found in http://oeis.org/A008303. Now, set

$$
\hat{B}_{n}(t):=\sum_{j \geq 0} \hat{b}_{n, j} t^{j}, \quad G(u, t):=\sum_{n \geq 0} \frac{\hat{B}_{n}(t)}{n !} u^{n} .
$$

In [29], it is proved that

$$
\hat{B}_{n}(t)=(2+t(n-2)) \hat{B}_{n-1}(t)+2 t(1-t) \hat{B}_{n-1}^{\prime}(t), \text { for } n \geq 2 .
$$

We prove a multivariate generalization of this recursion further down in Proposition 4.1. D. Warren and E. Seneta also proved that all zeros of $\hat{B}_{n}(t)$ are real and that $\hat{B}_{n-1} \ll \hat{B}(n)$ by using (16). We believe that a multivariate generalization of this holds, see Conjecture 4.1 below.

In $[14$, Cor. 23] it is shown that

$$
G(u, t)=-\frac{1}{t}+\frac{\sqrt{t-1}}{t} \tan (u \sqrt{t-1}+\arctan (1 / \sqrt{t-1}))=\frac{\tan (u \sqrt{t-1})}{\sqrt{t-1}-\tan (u \sqrt{t-1})},
$$

where the second follows from the first by using Mathematica.

The first few values of $\hat{B}_{n}(t)$ are presented in Table 3 .

\begin{tabular}{rr}
$n$ & $\hat{B}_{n}(t)$ \\
\hline 1 & 1 \\
2 & 2 \\
3 & $2 t+4$ \\
4 & $16 t+8$ \\
5 & $16 t^{2}+88 t+16$ \\
6 & $272 t^{2}+416 t+32$ \\
\hline
\end{tabular}

Table 3: The polynomials $\hat{B}_{n}(t)$. For example, there are 272 permutations in $S_{6}$ with 2 peaks.

For the remainder of this subsection, we shall focus our attention on the multivariate polynomial

$$
\hat{B}_{n}(\mathbf{x}):=\sum_{\pi \in S_{n}} \mathbf{x}_{\mathrm{PKV}(\pi)}
$$

which generalizes $\hat{B}_{n}(t)$. Note that $\hat{B}_{n}(\mathbf{x})$ is multiaffine i.e., it is linear in each fixed variable. We shall use the shorthand $\partial_{x_{j}}:=\partial / \partial_{x_{j}}$.

For $\pi \in S_{n-1}$ and $a \in[n-1]$, we let $\operatorname{Stay}_{a}(\pi)$ denote the permutation obtained from $\pi$ by inserting $n$ immediately after $a$. We also let $\operatorname{Stay}_{\emptyset}(\pi)$ denote the permutation obtained from $\pi$ by inserting $n$ before $\pi$ in one-line notation.

The following lemma allows us to track peaks when recursively constructing permutations. The motivation for the two sub-cases in (5) will be clear later in Lemma 4.7. For now, all arguments below do not need to distinguish the two sub-cases, unless stated explicitly. 
Lemma 4.1. For any $n \geq 1$, the bijection

$$
\mathcal{B}:\{\emptyset, 1,2, \ldots, n-1\} \times S_{n-1} \rightarrow S_{n}
$$

defined via $\mathcal{B}(a, \pi):=\operatorname{Stay}_{a}(\pi)$, has the following properties. For simplicity, we set $\pi^{\prime}:=\operatorname{Stay}_{a}(\pi)$ and we let $k$ be the value immediately succeeding a in $\pi$ (unless $a$ is the last entry in $\pi$ ).

1. $a=\emptyset$, so $\operatorname{PKV}\left(\pi^{\prime}\right)=\operatorname{PKV}(\pi)$.

2. $a$ is the last entry of $\pi$, so $\operatorname{PKV}\left(\pi^{\prime}\right)=\operatorname{PKV}(\pi)$.

3. $a \in \operatorname{PKV}(\pi)$. Then $\operatorname{PKV}\left(\pi^{\prime}\right)=(\operatorname{PKV}(\pi) \backslash\{a\}) \cup\{n\}$.

4. $k \in \operatorname{PKV}(\pi)$. Then $\operatorname{PKV}\left(\pi^{\prime}\right)=(\operatorname{PKV}(\pi) \backslash\{k\}) \cup\{n\}$.

5. $a$ is not the last entry of $\pi$, and neither $a$ or $k$ are in $\operatorname{PKV}(\pi)$. Then

(a) if a $<$, we have that $\operatorname{PKV}\left(\pi^{\prime}\right)=\operatorname{PKV}(\pi) \cup\{n\}$,

(b) otherwise $a>k$, and we also have that $\operatorname{PKV}\left(\pi^{\prime}\right)=\operatorname{PKV}(\pi) \cup\{n\}$.

Proof. First note that the map $\mathcal{B}$ is indeed a bijection, as we can easily recover $a$ from $\pi^{\prime}$, and $\pi$ is recovered from $\pi^{\prime}$ by removing $n$.

The other properties regarding the peaks follow via case-by-case analysis. We should perhaps clarify why the cases (3) and (4) are indeed mutually exclusive; two elements in $\operatorname{PKV}(\pi)$ cannot be adjacent in $\pi$.

Lemma 4.2. Let $\pi \in S_{n-1}$ and $m=|\mathrm{PKV}(\pi)|$ and $r=|\mathrm{DB}(\pi)|$. We can then count how many values of $a \in\{\emptyset, 1,2, \ldots, n-1\}$, belong to each case in Lemma 4.1.

1. For Case 1, there is only one option;

2. For Case 2, there is only one option;

3. For Case 3, there are $m$ options;

4. For Case 4, there are $m$ options;

5. For Case 5a, there are $(n-2)-r-m$ options;

6. For Case $5 b$, there are $r-m$ options.

Proof. Cases 1-4 follow directly from the the definition of the cases, e.g., we can insert $n$ after each peak value, so there are $m$ options in Case 3.

Moreover, if we add all options listed, we get $n$ (as expected), so it suffices to show that the number of options in Case 5a is $(n-2)-r-m$.

Consider a permutation $\pi \in S_{n-1}$. Then there are a total of $n-2$ spots where $n$ can be inserted between two entries. However, there are $m=|\operatorname{PKV}(\pi)|$ forbidden spots, as we are not allowed to insert $n$ before a peak. Moreover, we are not allowed to insert $n$ between $a$ and $k$, if $a>k$, as this is Case $5 \mathrm{~b}$. There are exactly $r$ such occurrences of $a$. Hence, what remains are $(n-2)-r-m$ valid options of $a$, where $a<k$.

As a corollary of Lemma 4.1, we can now easily deduce (15), and prove the following multivariate generalization of (16).

Proposition 4.1. We have that the multivariate polynomials $\hat{B}_{n}(\mathbf{x})$ satisfy the recursion

$$
\hat{B}_{n}=\left(2+(n-2) x_{n}\right) \hat{B}_{n-1}+2 x_{n} \sum_{j=3}^{n}\left(1-x_{j}\right) \cdot \partial_{x_{j}} \hat{B}_{n-1} .
$$

Proof. We shall prove that the recursion in (19) has the same structure as the recursion described in Lemma 4.1. We rewrite (19) as

$$
\begin{aligned}
\hat{B}_{n} & =\underbrace{\hat{B}_{n-1}}_{\text {Case } 1}+\underbrace{\hat{B}_{n-1}}_{\text {Case } 2}+\underbrace{x_{n} \sum_{a=3}^{n} \partial_{x_{a}} \hat{B}_{n-1}}_{\text {Case } 3}+\underbrace{x_{n} \sum_{k=3}^{n} \partial_{x_{k}} \hat{B}_{n-1}}_{\text {Case } 4} \\
& +\underbrace{x_{n}\left[(n-2) \hat{B}_{n-1}-x_{a}\left(\sum_{a=3}^{n} \partial_{x_{a}} \hat{B}_{n-1}\right)-x_{k}\left(\sum_{k=3}^{n} \partial_{x_{k}} \hat{B}_{n-1}\right)\right]}_{\text {Case } 5} .
\end{aligned}
$$


It remains to show that the equation labels indeed correspond to the five cases in Lemma 4.1. That $\hat{B}_{n-1}$ corresponds to cases 1 and 2 is evident.

In Case 3, we use $\partial_{x_{a}}$ to select all permutations counted by $\hat{B}_{n-1}$ where $a$ is a peak. This peak is then replaced by inserting $n$ after $a$, which makes $n$ a new peak. A similar argument applies in Case 4 .

In the last case, for any permutation of length $n-1$, there are $n-2$ potential spots where we may insert $n$ to make a peak, and a new permutation $\pi \in S_{n}$. However, we do not want to include the cases where a previous peak is replaced as these are covered by Case 3 and 4 . We, therefore, want to exclude the permutations counted by $\hat{B}_{n-1}$, where the entry immediately before $n$ in $\pi$ was a peak, or the entry immediately after $n$ was a peak.

As mentioned earlier, it has been shown that the $\hat{B}_{n}(t)$ are real-rooted. Computer experiments suggest the following stronger property for the multivariate version.

Conjecture 4.1. The polynomials $\hat{B}_{n}(\mathbf{x})=\sum_{\pi \in S_{n}} \mathbf{x}_{\mathrm{PKV}(\pi)}$, are all stable. Moreover, of $\lambda_{1}, \lambda_{2}, \ldots$ is a fixed sequence of positive real numbers, then

$$
\hat{B}_{n-1}\left(\lambda_{1} t, \lambda_{2} t, \ldots, \lambda_{n-1} t\right) \ll \hat{B}_{n}\left(\lambda_{1} t, \lambda_{2} t, \ldots, \lambda_{n} t\right) \text { for all } n>1 .
$$

A family of multi-affine polynomials of similar flavor is considered in [2, p.10], which are shown to be Hurwitz stable.

\subsection{A recursion which tracks peak-values after run-sort}

We have a recursive structure of permutations and peak-values described in Lemma 4.1. Our goal is now to describe an analogous recursive structure for permutations, but now keeping track of the set of peak-values after run-sorting. Again we keep track of the position where $n$ is inserted and have five cases corresponding to the cases in Lemma 4.1. However, some operations are more complicated, and we employ some operations on runs that are reminiscent of crossover on strands of DNA. A rough overview of our approach is given in Figure 1.

\begin{tabular}{|l|}
\hline Case 1 \\
\hline Case 2 \\
\hline Case 3 \\
\hline Case 4 \\
\hline Non-a-peak-admissible \\
\hline Case $5 a / b$ \\
\\
\hline Swap-image \\
\hline \\
\hline $\mathcal{E}^{1}$ \\
\hline $\mathcal{E}^{2}$ \\
\hline $\mathcal{E}^{3}$ \\
\hline $\mathcal{E}^{4}$ \\
\hline $\mathcal{E}^{5}$ \\
\hline
\end{tabular}

Figure 1: Cases 1, 2 and 3 are easy to handle, i.e., by simply using Stay ${ }_{a}$ on the permutation. In the remaining cases, we use Stay ${ }_{a}$ whenever it has the desired effect. Otherwise, we do some modifications. This modification has to be compensated for later. This leads to a handful of involutions that cancel the cases not handled by Stay $_{a}$. In the figure, two shaded sets of the same size and color are put in bijective correspondence via such an involution. The notation and terminology are explained later in this section.

Let $\operatorname{SPV}(\pi):=\operatorname{PKV}(\operatorname{runsort}(\pi))$ be the set of peak-values obtained after run-sorting $\pi$. In this subsection, we shall mainly consider the runs of $\pi \in S_{n-1}$ arranged in lexicographical order. We illustrate the runs of $\pi$ as 
shown in (20), where the $s_{i}$ are the start of the runs, with $s_{1}<s_{2}<\cdots<s_{r}$ :

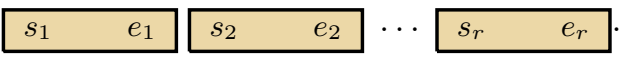

By definition, $\operatorname{SPV}(\pi) \subseteq\left\{e_{1}, e_{2}, \ldots, e_{r-1}\right\}$. Note that it is not guaranteed that $e_{i}>s_{i+1}$ for all $i \in[r-1]$, since (20) lists the runs of $\pi$, not the runs of runsort $(\pi)$. In this context, when referring to the runs of $\pi$, the words last, next, etc., are with respect to the ordering in (20), and not the order in which they appear in $\pi$.

From now on, we have a fixed $\pi \in S_{n-1}$ and $a \in\{1,2, \ldots, n-1\}$ present in the context we are working in. Let $\alpha$ denote the run of $\pi$ containing $a$ and let $k$ be the value immediately succeeding $a$, if such an entry exist. Furthermore, $\beta$ is the run succeeding $\alpha$ (in lex-order) and $\omega$ is the last run of $\pi$. Now, either $a$ and $k$ are both in $\alpha$, or $a$ is the largest value in $\alpha$. In the latter case, either $\alpha$ is not the last run, and $k$ is the smallest entry in $\beta$, or $a$ is simply the very last entry in $\operatorname{runsort}(\pi)$, see Equations (21) to (23).

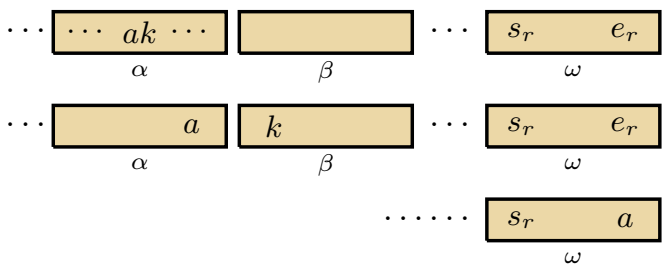

Suppose now $a$ is the penultimate entry in $\alpha$ and thus $k$ is the largest entry in $\alpha$ :

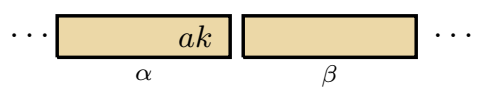

In the case $k \in \operatorname{SPV}(\pi)$, we say that $\pi$ is a-peak-admissible if

$$
\operatorname{SPV}\left(\operatorname{Stay}_{a}(\pi)\right)=(\operatorname{SPV}(\pi) \backslash\{k\}) \cup\{n\},
$$

that is, inserting $n$ after $a$, replaces $k$ by $n$ as a peak value after run-sorting. (Recall that $\operatorname{Stay}_{a}(\pi)$ was defined as the permutation obtained from $\pi$ by inserting $n$ immediately after $a$.)

Example 4.1. For example, consider

$$
\sigma=52674318 \text {, and } \pi=38256714 \text { which lie in } S_{8} .
$$

We have that $\operatorname{SPV}(\sigma)=\{7,8\}$ and $\operatorname{SPV}(\pi)=\{4,7\}, \operatorname{Stay}_{6}(\sigma)=526974318$ and $\operatorname{Stay}_{6}(\pi)=382569714$, $\operatorname{SPV}\left(\operatorname{Stay}_{6}(\sigma)\right)=\{8,9\}=(\operatorname{SPV}(\sigma) \backslash\{7\}) \cup\{9\}, \operatorname{SPV}\left(\operatorname{Stay}_{6}(\pi)\right)=\{4,8,9\} \neq(\operatorname{SPV}(\pi) \backslash\{7\}) \cup\{9\}$.

Hence, $\sigma$ is 6-peak-admissible, but $\pi$ is not.

The following lemma characterizes the $a$-peak-admissible permutations.

Lemma 4.3. Let $\pi \in S_{n-1}$ be a permutation with $k \in \mathrm{SPV}(\pi)$, and let a be the entry immediately before $k$ :

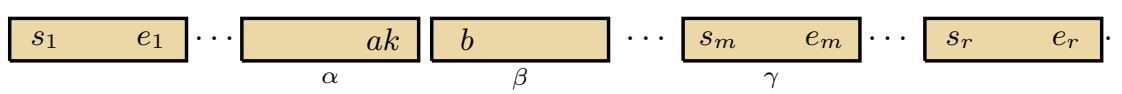

Let $m \in[r]$ be the largest value such that $s_{m}<k$. Then $\pi$ is a-peak-admissible if and only if

(i) $e_{m}<k$, or

(ii) $m<r$ and $e_{m}>s_{m+1}$.

Moreover, if $\pi$ is not a-peak-admissible, then $s_{m}<k<e_{m}<s_{m+1}$ and

$$
\operatorname{SPV}\left(\operatorname{Stay}_{a}(\pi)\right)=(\operatorname{SPV}(\pi) \backslash\{k\}) \cup\left\{e_{m}, n\right\} .
$$

Proof. First, it is clear that the number $m$ exists; since $k \in \operatorname{SPV}(\pi)$, we know that $b<k$, so the set of runs where the first entry is less than $k$ is non-empty.

Note that by the choice of $m$, we have additional inequalities in the two cases:

(i) $e_{m}<k<s_{m+1}$, or

(ii) $m<r$ and $k<s_{m+1}<e_{m}$. 
Suppose $\pi$ is $a$-peak-admissible and let $\pi^{\prime}=\operatorname{Stay}_{a}(\pi)$.

Consider the lex-largest run of $\pi^{\prime}$, where the starting point $s_{m}<k$. We have two cases to consider here i.e., whether $m=r$ or $m<r$. (i) If $m=r$, then $k$ appears at the end of $\pi^{\prime}$ and hence will not be an element in $\operatorname{SPV}\left(\pi^{\prime}\right)$. Otherwise if $r<m$ and $k<s_{m+1}$, then $k$ appears immediately after $\gamma$ in $\pi^{\prime}$ and before $s_{m+1}$ in $\pi^{\prime}$. Hence, $k$ is again not a peak-value of $\operatorname{runsort}\left(\pi^{\prime}\right)$. Moreover, $e_{m} \notin \operatorname{SPV}(\pi)$ and we still have $e_{m} \notin \operatorname{SPV}\left(\pi^{\prime}\right)$.

(ii) Since $m<r$, we know that $k<s_{m+1}$. Moreover, since $e_{m}>s_{m+1}$ we have that $e_{m} \in \operatorname{SPV}(\pi)$. Again, $k$ appears immediately after $e_{m}$ in $\operatorname{runsort}\left(\pi^{\prime}\right)$ and we still have $e_{m} \in \operatorname{SPV}\left(\pi^{\prime}\right)$. Hence, we have now shown that $\pi$ is $a$-peak-admissible in both cases listed.

It is now straightforward to verify that the condition $s_{m}<k<e_{m}<s_{m+1}$ is precisely the complement of the events (i) and (ii) above. Thus, we only need to show that $\operatorname{SPV}\left(\operatorname{Stay}_{a}(\pi)\right)=(\operatorname{SPV}(\pi) \backslash\{k\}) \cup\left\{e_{m}, n\right\}$, whenever $s_{m}<k<e_{m}<s_{m+1}$. But from these inequalities, we can deduce that $e_{m} \notin \operatorname{SPV}(\pi)$ and that the runs of $\operatorname{Stay}_{a}(\pi)$ are arranged lexicographically as

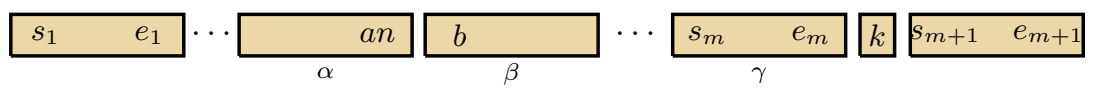

It is now evident that $e_{m} \in \operatorname{SPV}\left(\operatorname{Stay}_{a}(\pi)\right)$, and that (26) is true.

Definition 4.1. Suppose now that $\pi \in S_{n-1}, k \in \mathrm{SPV}(\pi)$ and a preceding $k$, but $\pi$ is not a-peak-admissible. We then construct a permutation denoted $\operatorname{Swap}_{a}(\pi)$ via the following procedure.

(i) Let

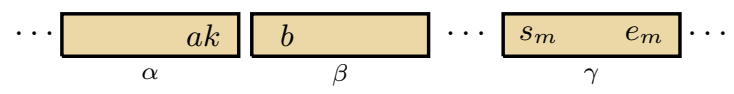

be the runs of $\pi$, as in Lemma 4.3 .

(ii) Let $\gamma$ be the largest run with $s_{m}<k<e_{m}$ (there is such a run, according to Lemma 4.3).

(iii) We split $\gamma$ as $\gamma_{1} \gamma_{2}$, where $\gamma_{1}$ is the prefix of $\gamma$ consisting of the elements which are less than $k$, while $\gamma_{2}$ are the remaining elements.

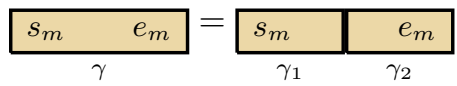

We let $\operatorname{Swap}_{a}(\pi)$ be the permutation obtained from $\pi$ by moving the elements in $\gamma_{2}$, and inserting them immediately after $k$. Hence, the lex-sorted runs of $\operatorname{Swap}_{a}(\pi)$ are

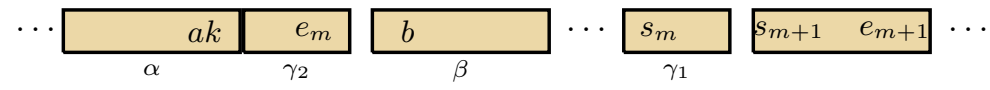

The run $\gamma$ does not need to be located somewhere after $\alpha$ in $\pi$, it can be located anywhere in $\pi$. The run immediately after $\gamma_{1}$ in $\operatorname{Swap}_{a}(\pi)$, (let's call it $\delta$ ) must start with something less than $e_{m}$ since otherwise, $e_{m}$ would not be the largest value in its run. Moreover, since $s_{m+1}$ is the smallest starting value of a run greater than $k$, and $s_{m+1}>e_{m}$ (because $\pi$ is non-a-peak-admissible), it follows that the first entry of $\delta$ must be less than $s_{m}$. This implies that $\gamma_{1}$ does not 'merge' with $\delta$, as $\gamma_{2}$ is moved to a different part of $\pi$. In other words, $\gamma_{1}$ is still a run in $\operatorname{Swap}_{a}(\pi)$. The runs of $\operatorname{Stay}_{a}\left(\operatorname{Swap}_{a}(\pi)\right)$ in lexicographical order are therefore

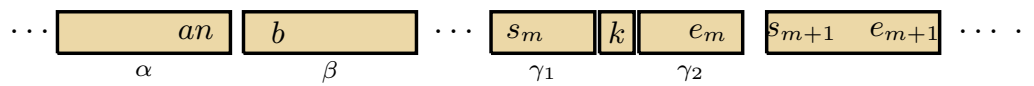

Example 4.2. We use the same notation as in the description of $\mathrm{Swap}_{a}$. Let $\pi=38256714$ and $a=6, k=7$. The runs of $\pi$ in lex-order are 14256738 . Then $\gamma=38$ and $\gamma_{1}=3, \gamma_{2}=8$. Hence, $\operatorname{Swap}_{a}(\pi)=32567814$ because 8 is moved and inserted after $k=7$.

Lemma 4.4. Let $D_{n}$ be the subset of pairs $(a, \pi) \in[n-1] \times S_{n-1}$ such that a immediately precedes an entry in $\operatorname{SPV}(\pi)$, but $\pi$ is not a-peak-admissible. Then the map from $D_{n}$ to $S_{n}$ defined by

$$
(a, \pi) \mapsto \operatorname{Stay}_{a}\left(\operatorname{Swap}_{a}(\pi)\right)
$$

is well-defined and injective. Moreover, for $(a, \pi) \in D_{n}$, we have that

$$
\operatorname{SPV}\left(\operatorname{Stay}_{a}\left(\operatorname{Swap}_{a}(\pi)\right)\right)=(\operatorname{SPV}(\pi) \backslash\{k\}) \cup\{n\} .
$$


Proof. Since $\operatorname{Swap}_{a}(\pi)$ is well-defined for $(a, \pi) \in D_{n}$, it follows that the map in (31) is also well-defined. Now, given the output of $\mathrm{Stay}_{a}$, we can recover $a$ (it is the entry immediately to the left of $n$ ) and thus $k$. It now suffices to show that $\operatorname{Swap}_{a}$ is injective on the set of permutations that are not $a$-peak-admissible. However, given the output of $\operatorname{Swap}_{a}(\pi)$ for some known $a$, we can identify $k$ and then also $\gamma_{2}$ for $\pi$. Now $\gamma_{1}$ is the lex-largest run whose first entry is less than $k$. It is now clear that we can recover $\pi$ from $\operatorname{Stay}_{a}\left(\operatorname{Swap}_{a}(\pi)\right)$. The fact that (32) holds follows from comparing (28) with (30) - it is evident that $n$ is a peak-value, and that $k$ is now a starting point of a run in $\pi$ (so in particular, not a peak-value). All other peak-values after run-sort are preserved.

Definition 4.2 (Swap-image). If there is some non-a-peak-admissible $\sigma \in S_{n-1}$ such that $\sigma=\operatorname{Swap}_{a}(\pi)$, we say that $\pi \in S_{n-1}$ is in the swap-image.

The permutations which are not in the swap-image, are handled later in this section.

Next, we treat the analog of the last case in Lemma 4.1, where we insert $n$ in such a way that it creates a new peak. For $\pi \in S_{n-1}$, let $\mathcal{S}(\pi)$ be the values of $a \in[n-1]$ such that if we insert $n$ after $a$ in $\operatorname{runsort}(\pi), n$ becomes a peak-value and all other peak values are preserved ${ }^{\S}$. If we let $\left(\pi_{1}^{\prime}, \pi_{2}^{\prime}, \ldots, \pi_{n-1}^{\prime}\right):=\operatorname{runsort}(\pi)$, then $\mathcal{S}(\pi)$ can be described more explicitly as

$$
\mathcal{S}(\pi)=\left\{a: \operatorname{SPV}\left(\operatorname{Stay}_{a}(\pi)\right)=\operatorname{SPV}(\pi) \cup\{n\}\right\}=\left\{\pi_{i}^{\prime}: i \leq n-2, \pi_{i}^{\prime} \notin \operatorname{SPV}(\pi), \text { and } \pi_{i+1}^{\prime} \notin \operatorname{SPV}(\pi)\right\} .
$$

Example 4.3. Some permutations and their sets $\mathcal{S}(\pi)$ are shown below.

\begin{tabular}{lll} 
Permutation $\pi$ & $\operatorname{runsort}(\pi)$ & $\mathcal{S}(\pi)$ \\
\hline 2561734 & 1725634 & $\{2,3\}$ \\
4567123 & 1234567 & $\{1,2,3,4,5,6\}$ \\
4371625 & 1625374 & \{\} \\
\hline
\end{tabular}

We say that $\pi$ is a-slope-admissible if

$$
a \in \mathcal{S}(\pi) \text { and } \operatorname{SPV}\left(\operatorname{Stay}_{a}(\pi)\right)=\operatorname{SPV}(\pi) \cup\{n\}
$$

The next lemma contains additional technical results needed later for the main conclusion. We characterize the $a$-slope-admissible permutations and show that these are never in the swap-image. We also describe all non- $a$-slope-admissible permutations which are not in the swap-image.

Lemma 4.5. Let $\pi \in S_{n-1}$ be a permutation with $a \in \mathcal{S}(\pi)$ and write $\alpha$ as $\alpha_{1}$ a $\alpha_{2}$ :

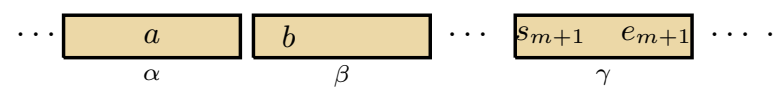

In the case $\alpha_{2} \neq \emptyset$, let $k$ and $l$ be the smallest and largest values in $\alpha_{2}$, respectively. Moreover, let $m \in[r-1]$ be the smallest value where $s_{m+1}>k$, if such an $m$ exists. With all these definitions, the runs of $\pi$ are as follows:

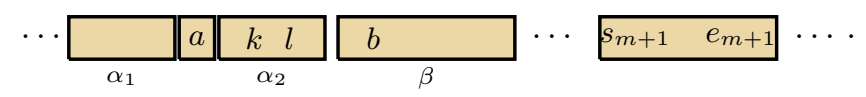

Statement 1: We have that $\pi$ is a-slope-admissible if and only if

(i) $\alpha_{2}=\emptyset$, or

(ii) $\alpha$ is the lex-largest run, or

(iii) $k<b$, or

(iv) $m$ exists, $l>s_{m+1}$ and $e_{m}<k \Longleftrightarrow e_{m}<s_{m+1}$.

Statement 2: We have that $\pi$ is not a-slope-admissible if and only if

(i') $\alpha_{2}$ is non-empty,

(ii') $\alpha$ is not the last run (so b exists),

\footnotetext{
$\S$ Note: Inserting $n$ does not preserve being run-sorted in general.
} 
(iii') $b<k$ and

(iv') either $m$ does not exist, or $l<s_{m+1}$ or $k<e_{m}<s_{m+1}$.

Statement 3: If $\pi$ is in the swap-image, then $\pi$ is not a-peak-admissible.

Statement 4: if $\pi$ is not a-peak-admissible and not in the swap-image, then one of the following conditions hold:

1. $m$ does not exist, (so $s_{j}<k$ for all $\left.j \in[r]\right), e_{r}>k$ and $\operatorname{SPV}\left(\operatorname{Stay}_{a}(\pi)\right)=(\operatorname{SPV}(\pi) \backslash\{l\}) \cup\left\{e_{r}, n\right\}$.

2. $k<l<e_{m}<s_{m+1}$, and $\operatorname{SPV}\left(\operatorname{Stay}_{a}(\pi)\right)=(\operatorname{SPV}(\pi) \backslash\{l\}) \cup\left\{e_{m}, n\right\}$.

3. $k<e_{m}<l<s_{m+1}$, and $\operatorname{SPV}\left(\operatorname{Stay}_{a}(\pi)\right)=(\operatorname{SPV}(\pi) \backslash\{l\}) \cup\left\{e_{m}, n\right\}$.

4. $k<l<s_{m+1}<e_{m}$, and $\operatorname{SPV}\left(\operatorname{Stay}_{a}(\pi)\right)=(\operatorname{SPV}(\pi) \backslash\{l\}) \cup\{n\}$.

5. $k<e_{m}<s_{m+1}<l$, and $\operatorname{SPV}\left(\operatorname{Stay}_{a}(\pi)\right)=\operatorname{SPV}(\pi) \cup\left\{e_{m}, n\right\}$.

Proof. Proof of statement 1: We first verify that cases (i)-(iv) are indeed $a$-slope-admissible. The first three cases are easy, since inserting $n$ after $a$ splits $\alpha$ into two smaller runs, but the two resulting runs are adjacent in the lex-order, as in (35). Thus, only case (iv) requires some more work.

So first we suppose that $l>s_{m+1}$ and that both $e_{m}<k$ and $e_{m}<s_{m+1}$ hold, i.e., $e_{m}$ is not a peak-value. Then, inserting $n$ after $a$ in (35), gives that $\operatorname{Stay}_{a}(\pi)$ has the runs

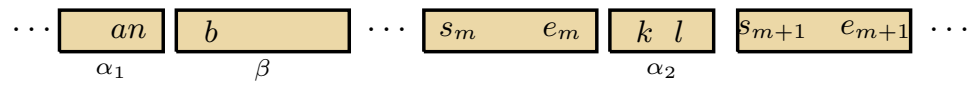

Since $l>s_{m+1}$, we still have that $l$ is a peak-value, and $e_{m}<k$ implies that $e_{m}$ is still not a peak-value. Hence, $\pi$ is $a$-peak-admissible. The second sub-case, when $l>s_{m+1}$ but both $e_{m}<k$ and $e_{m}<s_{m+1}$ are false, is treated in a similar manner.

Proof of statement 2: We now show that $\pi$ is not $a$-slope-admissible if $\alpha_{2} \neq \emptyset, b<k$ and one of the cases
(a) $m$ does not exist,
(b) $\quad l<s_{m+1}$
(c) $k<e_{m}<s_{m+1}$

hold.

In case (a), we have that $s_{j}<k$ for all $j \in[r]$, so the runs of $\operatorname{Stay}_{a}(\pi)$ in lex-order are

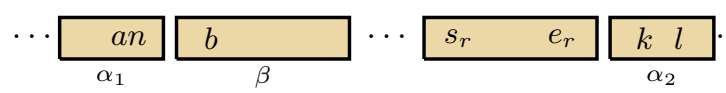

Hence,

$$
\operatorname{SPV}\left(\operatorname{Stay}_{a}(\pi)\right)= \begin{cases}(\operatorname{SPV}(\pi) \backslash\{l\}) \cup\{n\} & \text { if } e_{r}<k \\ (\operatorname{SPV}(\pi) \backslash\{l\}) \cup\left\{e_{r}, n\right\} & \text { if } e_{r}>k\end{cases}
$$

and neither case is therefore $a$-peak-admissible.

In cases (b) and (c), we get the situation illustrated in (36), but with

$$
\operatorname{SPV}\left(\operatorname{Stay}_{a}(\pi)\right)= \begin{cases}(\operatorname{SPV}(\pi) \backslash\{l\}) \cup\{n\} & \text { if } e_{m}<k, e_{m}<s_{m+1}, l<s_{m+1} \\ (\operatorname{SPV}(\pi) \backslash\{l\}) \cup\{n\} & \text { if } e_{m}>k, e_{m}>s_{m+1}, l<s_{m+1} \\ (\operatorname{SPV}(\pi) \backslash\{l\}) \cup\left\{e_{m}, n\right\} & \text { if } k<e_{m}<s_{m+1}, l<s_{m+1} \\ \operatorname{SPV}(\pi) \cup\left\{e_{m}, n\right\} & \text { if } k<e_{m}<s_{m+1}, l>s_{m+1} .\end{cases}
$$

In order for $\pi$ to be $a$-slope-admissible, we must still have $l$ as a peak-value, and $n$ as the only additional peak-value-this is not the case in any of the outcomes.

Proof of statement 3: We shall now show that if $\pi$ is in the swap-image, then $\pi$ is not $a$-slope-admissible.

Suppose $\pi=\operatorname{Swap}_{a}(\sigma)$ for some non- $a$-peak-admissible $\sigma \in S_{n-1}$. The runs of $\pi$, according to (29), are

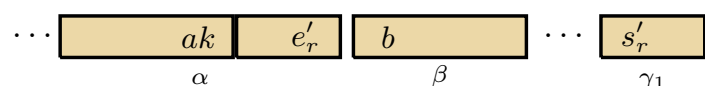

or 


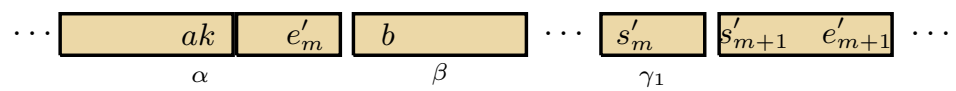

where $e_{m}^{\prime}<s_{m+1}^{\prime}$ and the last entry of $\gamma_{1}$ is less than $k$. We now must verify conditions (i')-(iv') for $\pi$. The first three conditions are easy to verify. Now, if $\gamma_{1}$ is the last run of $\pi$, we have that case (a) of (iv') is satisfied (there is no run of $\pi$ where the first value exceeds $k$ ).

If $\gamma_{1}$ is not the last run, we know that $e_{m}^{\prime}<s_{m+1}^{\prime}$, so $l=e_{m}^{\prime}<s_{m+1}^{\prime}=s_{m+1}$ and thus the condition in case (b) is satisfied.

Proof of statement 4: We shall now refine the previous and see exactly what is possible for $\pi$ being non- $a$ slope-admissible and also not in the swap-image.

A more careful case-by-case analysis as before, shows that $\pi$ is in the swap-image only if it satisfies the first of the four cases listed in (b)-(c). We can then conclude Statement 4.

Let $\mathcal{E}_{n, a}^{j}$ be the set of permutations $\pi \in S_{n-1}$ such that

- $a \in \mathcal{S}(\pi)$,

- $\pi$ is not $a$-slope-admissible, and

- $\pi$ satisfies Case $(j)$ of Statement 4 in Lemma 4.5.

Note that $\mathcal{E}_{n, a}^{1}, \ldots, \mathcal{E}_{n, a}^{5}$ are disjoint sets.

Example 4.4. For example, for $n=7$ and $a=3$, we have that $\mathcal{E}_{n, a}^{1}$ comprises 40 permutations, including 1235647 and 4735621. Moreover, we have that

$$
\begin{array}{ll}
\mathcal{E}_{n, a}^{2}=\{7134526,7261345\}, & \mathcal{E}_{n, a}^{3}=\{7134625,7251346\}, \\
\mathcal{E}_{n, a}^{4}=\{1345276,2761345,6134527,6271345\}, & \mathcal{E}_{n, a}^{4}=\{1347625,2513476,6134725,6251347\} .
\end{array}
$$

We shall see below that $\left|\mathcal{E}_{n, a}^{2}\right|=\left|\mathcal{E}_{n, a}^{3}\right|$ and $\left|\mathcal{E}_{n, a}^{4}\right|=\left|\mathcal{E}_{n, a}^{5}\right|$ in general.

Definition 4.3. Suppose $\pi \in S_{n-1}$ belongs to some $\mathcal{E}_{n, a}^{j}$, and as always, let $k$ be the element succeeding a. We then know that there is at least one run $s_{m}, \ldots, e_{m}$ of $\pi$, such that $s_{m}<k<e_{m}$, so choose $m$ which maximizes $s_{m}$. Hence, the runs of $\pi$ are

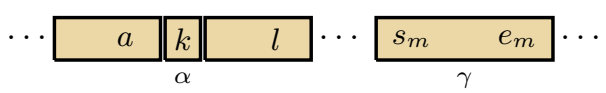

where $l>k$ is the largest entry in the run containing a. As in the construction of $\mathrm{Swap}_{a}$, we write the $\gamma$-run as a concatenation of the elements less than $k$, and elements greater than $k$ :

$$
\begin{array}{|ll|}
\hline s_{m} & e_{m} \\
\hline
\end{array} \begin{array}{|l|l|}
\hline s_{m} & e_{m} \\
\hline
\end{array}
$$

Now, let $\mu$ denote the contiguous sequence of runs (possibly empty) appearing immediately after $l$ in $\pi$, starting with an element greater than $e_{m}$. Similarly, let $\nu$ be the contiguous sequence of runs (possibly empty) appearing immediately after $e_{m}$ in $\pi$, starting with an element greater than $l$.

Hence, the elements in $\alpha$ which are strictly greater than $k$, together with the elements in $\mu$ form a contiguous segment of elements in $\pi$. Let us call this segment $\Sigma_{1}$. Moreover, the elements in $\gamma$ greater than $k$, together with the elements in $\nu$ also form a contiguous segment, $\Sigma_{2}$. We then define $\operatorname{Flip}_{a}(\pi)$ as the permutation obtained from $\pi$ by interchanging $\Sigma_{1}$ and $\Sigma_{2}$. It is straightforward to verify that Flip $\mathrm{p}_{a}$ is an involution.

Finally, we note that the runs of $\operatorname{Stay}_{a}\left(\operatorname{Flip}_{a}(\pi)\right)$ are

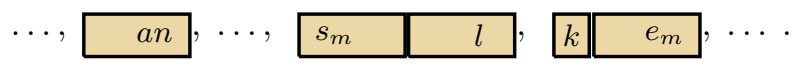

Example 4.5. Consider a permutation $\pi=83724561$, with $a=2$. Then $k=4$, and we have runs $\alpha=2456$ and $\gamma=37$. Moreover, $\mu_{1}=\nu_{2}=\emptyset$. So, $\operatorname{Flip}_{2}(\pi)$ is given by interchanging $\Sigma_{1}=56$ and $\Sigma_{2}=7$ in $\pi$, which gives $\operatorname{Flip}_{2}(\pi)=83562471$.

Consider now instead $\pi=134265$, with $a=1$. Here, $k=3, \mu=\emptyset$ and $\nu=5, \Sigma_{1}=4, \Sigma_{2}=65$. We have that $\operatorname{Flip}_{1}(\pi)=136524$.

Additional examples are shown in Table 4. 


\begin{tabular}{lccc}
$\pi$ & Case & $\operatorname{Flip}_{1}(\pi)$ & Case \\
\hline 2351467 & 1 & 2367145 & 1 \\
2571346 & 1 & 2461357 & 1 \\
1473526 & 1 & 1453726 & 1 \\
2467135 & 1 & 2513467 & 1 \\
\hline 7135246 & 2 & 7134625 & 3 \\
7134526 & 2 & 7136245 & 3 \\
7625134 & 2 & 7624135 & 3 \\
7362145 & 2 & 7352146 & 3 \\
\hline 1342576 & 4 & 1357624 & 5 \\
6134275 & 4 & 6137524 & 5 \\
2476135 & 4 & 2513476 & 5 \\
7265134 & 4 & 7241365 & 5 \\
1342756 & 4 & 1375624 & 5 \\
2657134 & 4 & 2413657 & 5 \\
3762145 & 4 & 3521476 & 5 \\
2761345 & 4 & 2451376 & 5 \\
\hline
\end{tabular}

Table 4: Action of Flip $_{1}$ on various members of $\mathcal{E}_{7,1}^{j}$, for different choices of $j \in\{1,2,4\}$ (second column). The last column tells us in which set $\mathcal{E}_{7,1}^{j}$ where $\operatorname{Flip}_{1}(\pi)$ belongs. As we see, this agrees with the statements in Lemma 4.6.

Lemma 4.6. The map Flip ${ }_{a}$, acts as follows:

$$
\begin{aligned}
& \operatorname{Flip}_{a}: \mathcal{E}_{n, a}^{1} \rightarrow \mathcal{E}_{n, a}^{1}, \\
& \operatorname{Flip}_{a}: \mathcal{E}_{n, a}^{2} \rightarrow \mathcal{E}_{n, a}^{3}, \\
& \operatorname{Flip}_{a}: \mathcal{E}_{n, a}^{4} \rightarrow \mathcal{E}_{n, a}^{5},
\end{aligned}
$$$$
\operatorname{Flip}_{a}^{-1}: \mathcal{E}_{n, a}^{3} \rightarrow \mathcal{E}_{n, a}^{2}
$$$$
\operatorname{Flip}_{a}^{-1}: \mathcal{E}_{n, a}^{5} \rightarrow \mathcal{E}_{n, a}^{4} \text {. }
$$

Moreover, for each map $\psi \in\left\{\operatorname{Flip}_{a}, \operatorname{Flip}_{a}^{-1}\right\}$ above,

$$
\operatorname{SPV}(\pi) \cup\{n\}=\operatorname{SPV}\left(\operatorname{Stay}_{a}(\psi(\pi))\right),
$$

on the relevant domain, and $\psi$ preserves the set of descent bottoms.

Proof. It is fairly straightforward to verify that the domain and range match, by examining the five cases in Statement 4 of Lemma 4.5. Moreover, we have that $\mu=\nu=\emptyset$ (as in the definition of Flip ${ }_{a}$ ) unless we act on some permutation in $\mathcal{E}_{n, a}^{4}$ or $\mathcal{E}_{n, a}^{5}$.

The case $\operatorname{Flip}_{a}: \mathcal{E}_{n, a}^{1} \rightarrow \mathcal{E}_{n, a}^{1}$ follows the same structure as the other two cases, so we only consider those.

We first verify $\operatorname{Flip}_{a}: \mathcal{E}_{n, a}^{2} \rightarrow \mathcal{E}_{n, a}^{3}$. Let $\pi \in \mathcal{E}_{n, a}^{2}$, where the runs are

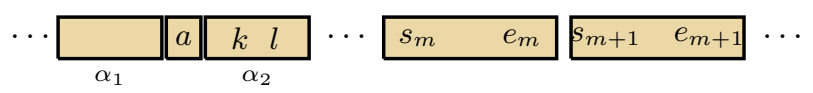

and we know that by the choice of $m$ and properties of $\mathcal{E}_{n, a}^{2}$, that $s_{m}<k<l<e_{m}<s_{m+1}$. Hence, $l \in \mathrm{SPV}(\pi)$ and $e_{m} \notin \operatorname{SPV}(\pi)$. Now as we saw in (40), the runs of $\pi^{\prime}:=\operatorname{Stay}_{a}\left(\operatorname{Flip}_{a}(\pi)\right)$ are

$$
\cdots \quad \cdots \quad \cdots \quad \begin{array}{l|l|l|l|l|l|l|l|l|l|l|}
\hline s_{m} & e_{m+1} & e_{m+1}
\end{array} \cdots
$$

We therefore have that $n, l \in \operatorname{SPV}\left(\pi^{\prime}\right)$ but $e_{m} \notin \operatorname{SPV}\left(\pi^{\prime}\right)$. All other peak-values are preserved, and it is verified that $\operatorname{SPV}(\pi) \cup\{n\}=\operatorname{SPV}\left(\operatorname{Stay}_{a}\left(\operatorname{Flip}_{a}(\pi)\right)\right)$.

Now for the case $\operatorname{Flip}_{a}: \mathcal{E}_{n, a}^{4} \rightarrow \mathcal{E}_{n, a}^{5}$. For $\pi \in \mathcal{E}_{n, a}^{2}$, the runs will satisfy $k<l<s_{m}<e_{m}<s_{m+1}$ and the exact same reasoning for the runs of $\pi$ is valid as in the previous case. However, now it is possible that Flip $_{a}$ does something involving non-empty $\mu$ and $\nu$ in Definition 4.3 , but by construction, this more complicated situation will still result in $\operatorname{Stay}_{a}\left(\operatorname{Flip}_{a}(\pi)\right)$ being of the form in (40). One can now verify again that $\operatorname{SPV}(\pi) \cup\{n\}=\operatorname{SPV}\left(\operatorname{Stay}_{a}\left(\operatorname{Flip}_{a}(\pi)\right)\right)$.

Finally, it is straightforward to see that the set of descent bottoms are preserved.

In Table 4, we illustrate the action of Flip $_{1}$ on some permutations in $\mathcal{E}_{7,1}^{j}$ for different choices of $j$.

The following result is now an analog of Lemma 4.1, and is the main result in this section. The reader is encouraged to review Figure 1 at this point. 
Theorem 4.1. For any $n \geq 1$, there is a bijection

$$
\mathcal{C}:\{\emptyset, 1,2, \ldots, n-1\} \times S_{n-1} \rightarrow S_{n}
$$

which has the following properties. For simplicity, we set $\pi^{\prime}:=\mathcal{C}(a, \pi)$ and we let $k$ be the value immediately succeeding a in $\operatorname{runsort}(\pi)$, unless $a$ is the last entry in $\operatorname{runsort}(\pi)$ :

1. $a=\emptyset$, and $\operatorname{SPV}\left(\pi^{\prime}\right)=\operatorname{SPV}(\pi)$.

2. $a$ is the last entry of $\operatorname{runsort}(\pi)$, and $\operatorname{SPV}\left(\pi^{\prime}\right)=\operatorname{SPV}(\pi)$.

3. $a \in \operatorname{SPV}(\pi)$. Then $\operatorname{SPV}\left(\pi^{\prime}\right)=(\operatorname{SPV}(\pi) \backslash\{a\}) \cup\{n\}$.

4. $k \in \operatorname{SPV}(\pi)$. Then $\operatorname{SPV}\left(\pi^{\prime}\right)=(\operatorname{SPV}(\pi) \backslash\{k\}) \cup\{n\}$.

5. $a$ is not the last entry of $\operatorname{runsort}(\pi)$, and neither $a$ or $k$ are in $\operatorname{SPV}(\pi)$. Then $\operatorname{SPV}\left(\pi^{\prime}\right)=\operatorname{SPV}(\pi) \cup\{n\}$.

Proof. We shall now describe how $\pi^{\prime}:=\mathcal{C}(a, \pi)$ is computed in each of the cases listed above.

Case 1: $a=\emptyset$ and $\pi^{\prime}=\operatorname{Stay}_{a}(\pi)$. It is straightforward to see that $\operatorname{SPV}\left(\pi^{\prime}\right)=\operatorname{SPV}(\pi)$.

Case 2: $a$ is the last entry of $\operatorname{runsort}(\pi)$ and $\pi^{\prime}=\operatorname{Stay}_{a}(\pi)$. Again, it is easy to verify that $\operatorname{SPV}\left(\pi^{\prime}\right)=\operatorname{SPV}(\pi)$.

In all remaining cases, $a$ appears in some run $\alpha$. Let $k$ be the entry immediately after $a$ in $\operatorname{runsort}(\pi)$. We note that at most one of $a$ and $k$ can be a member of $\operatorname{SPV}(\pi)$. In the case when $\alpha$ is not the last run, let $\beta$ be the run immediately succeeding $\alpha$.

Case 3: $a \in \operatorname{SPV}(\pi)$, and we know that $a>k$. We have that

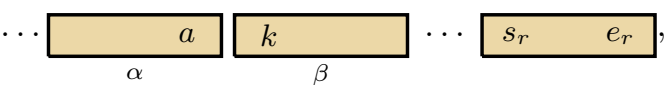

and we set $\pi^{\prime}=\operatorname{Stay}_{a}(\pi)$. The runs of $\pi$ can easily be identified with the runs of $\operatorname{Stay}_{a}(\pi)$, and hence

$$
\operatorname{SPV}\left(\pi^{\prime}\right)=(\operatorname{SPV}(\pi) \backslash\{a\}) \cup\{n\}
$$

Case 4: $k \in \operatorname{SPV}(\pi)$, so $\alpha$ is for sure not the lex-largest run of $\pi$. We are therefore in the situation

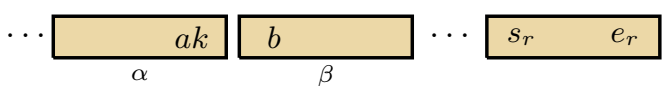

where $k>b$. If $\pi$ is $a$-peak-admissible, we let $\pi^{\prime}=\operatorname{Stay}_{a}(\pi)$, otherwise we set $\pi^{\prime}=\operatorname{Stay}_{a}\left(\operatorname{Swap}_{a}(\pi)\right)$. By definition of $a$-peak-admissible or by Lemma 4.4, we have that

$$
\operatorname{SPV}\left(\pi^{\prime}\right)=(\operatorname{SPV}(\pi) \backslash\{k\}) \cup\{n\} .
$$

Case 5: $a \notin P$ and $k \notin P$.

(a) If $\pi$ is $a$-slope-admissible, we set $\pi^{\prime}=\operatorname{Stay}_{a}(\pi)$. Note that by Lemma $4.5, \pi^{\prime}$ will not be equal to some $\operatorname{Stay}_{a}\left(\operatorname{Swap}_{a}(\sigma)\right)$, from Case 4 .

(b) If $\pi$ is in the swap-image, so that $\pi$ is of the form $\operatorname{Swap}_{a}(\sigma)$ for some (unique) $\sigma$, we let

$$
\pi^{\prime}=\operatorname{Stay}_{a}(\sigma)=\operatorname{Stay}_{a}\left(\operatorname{Swap}_{a}^{-1}(\pi)\right)
$$

To elaborate more on this choice, suppose that the runs of $\operatorname{Stay}_{a}(\pi)$ are of the form of $\operatorname{some~} \operatorname{Stay}_{a}\left(\operatorname{Swap}_{a}(\sigma)\right)$ described earlier in (29):

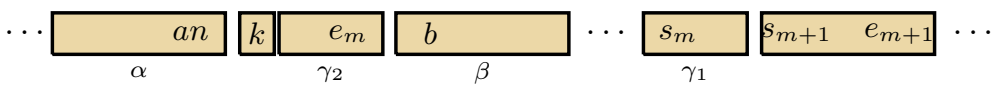

This means that $\operatorname{runsort}(\pi)$ is of the form

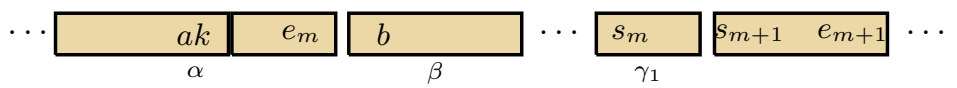


where $a, k$ and $e_{m}$ are in the same run, and $e_{m} \in \operatorname{SPV}(\pi)$. We can compute $\sigma=\operatorname{Swap}_{a}^{-1}(\pi)$ and the runs of $\sigma$ are

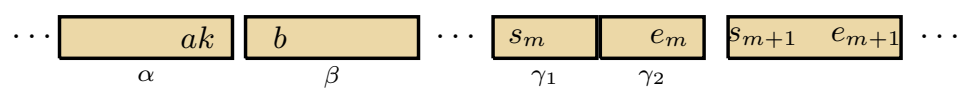

Finally, the runs of $\operatorname{Stay}_{a}(\sigma)$ are lexicographically sorted as

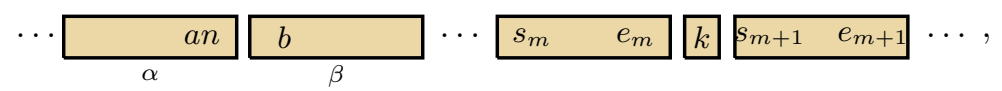

and it is now evident that $\operatorname{SPV}\left(\pi^{\prime}\right)=\operatorname{SPV}(\pi) \cup\{n\}$, as desired.

(c) The only remaining case is when $\pi \in \mathcal{E}_{n, a}^{1} \cup \cdots \cup \mathcal{E}_{n, a}^{5}$. We then let $\pi^{\prime}=\operatorname{Flip}_{a}(\pi)$, and by Lemma 4.6, we again have that $\operatorname{SPV}\left(\pi^{\prime}\right)=\operatorname{SPV}(\pi) \cup\{n\}$.

In each case, we can easily determine $a$ from $\pi^{\prime}$, as this is the entry to the left of $n$. All the non-trivial maps used, i.e., Flip $a$ and $\operatorname{Swap}_{a}$, are invertible, so it follows that the description above indeed defines a bijection.

The bijections $\mathcal{B}$ and $\mathcal{C}$ behave in a similar manner on other combinatorial statistics. Now we do need the distinction between Case $5 \mathrm{a}$ and Case $5 \mathrm{~b}$ (separating these cases is compatible with Flip ${ }_{a}$, since by Lemma 4.6 , Flip $_{a}$ preserves the set of descent bottoms).

In what follows, by convention, if $a=\emptyset$, set $k:=\pi_{1}$. We refer to the introduction where all permutation statistics are defined.

Lemma 4.7. Let $\sigma \in S_{n-1}$ and let $\sigma^{\prime}:=\mathcal{B}(\sigma, a)$ for $a \in\{\emptyset, 1,2, \ldots, n-1\}$ (and we let $k$ be the element succeeding a in $\sigma$ ). Then for each of the cases in Lemma 4.1, we have the following recursive relations:

\begin{tabular}{lllll} 
Case & $\sigma_{1}^{\prime}$ & $\operatorname{DB}\left(\sigma^{\prime}\right)$ & $\operatorname{LRMin}\left(\sigma^{\prime}\right)$ & $\operatorname{PKV}^{*}\left(\sigma^{\prime}\right)$ \\
\hline 1 & $n$ & $\operatorname{DB}(\sigma) \cup\{k\}$ & $\operatorname{LRMin}(\sigma) \cup\{n\}$ & $\operatorname{PKV}^{*}(\sigma)$ \\
2 & $\sigma_{1}$ & $\operatorname{DB}(\sigma)$ & $\operatorname{LRMin}(\sigma)$ & $\operatorname{PKV}^{*}(\sigma)$ \\
3 & $\sigma_{1}$ & $\operatorname{DB}(\sigma)$ & $\operatorname{LRMin}(\sigma)$ & $\operatorname{PKV}^{*}(\sigma)$ \\
4 & $\sigma_{1}$ & $\operatorname{DB}(\sigma) \cup\{k\}$ & $\operatorname{LRMin}(\sigma)$ & $\operatorname{PKV}^{*}(\sigma)$ \\
$5 a$ & $\sigma_{1}$ & $\operatorname{DB}(\sigma) \cup\{k\}$ & $\operatorname{LRMin}(\sigma)$ & $\left(\operatorname{PKV}^{*}(\sigma) \backslash\{a\}\right) \cup\{n\}$ \\
$5 b$ & $\sigma_{1}$ & $\operatorname{DB}(\sigma)$ & $\operatorname{LRMin}(\sigma)$ & $\operatorname{PKV}^{*}(\sigma) \backslash\{a\}$ \\
\hline
\end{tabular}

The same identities hold in the corresponding cases if we instead take $\sigma^{\prime}:=\mathcal{C}(\sigma, a)$.

Proof sketch. We need to examine both $\mathcal{B}(\cdot, a)$ and $\mathcal{C}(\cdot, a)$, and make sure that they affect the statistic in the same manner for each of the listed cases above.

Observation: Note that $k$ is always the number succeeding $n$ in $\sigma^{\prime}$, for both $\mathcal{B}(\cdot, a)$ and $\mathcal{C}(\cdot, a)$. To show this, one needs to go through all cases in Theorem 4.1, but it suffices to verify that $\operatorname{Swap}_{a}(\sigma)$ and Flip $a(\sigma)$ always have $k$ succeeding $a$.

First entry statistic. It is clear that if we insert $n$ after any entry $a$, the very first entry of the permutation is preserved. This is the case also for the more complicated map $\mathcal{C}(\cdot, a)$. Likewise, inserting $n$ in the very beginning makes it the first entry.

Descent bottom statistic. It is straightforward to verify the effect on the set of descent-bottoms when we simply apply $\operatorname{Stay}_{a}(\sigma)$. So then it remains to verify the same five cases for $\mathcal{C}(\sigma, a)$, but this follows more or less directly from the observation above.

Now, together with Lemma 4.2 , and the fact that the behavior of descent bottoms under $\mathcal{C}(\cdot, a)$ agrees with $\mathcal{B}(\cdot, a)$, implies that the number of values of $a$ belonging to Case $5 \mathrm{a}$, is the same for both maps.

Left to right minima statistic. For $\mathcal{B}(\cdot, a)$, we note that inserting $n$ somewhere in $\sigma$ does not affect the left to right minima, except when $n$ is inserted in the very beginning.

Now for $\mathcal{C}(\cdot, a)$, note that the maps $\operatorname{Swap}_{a}(\sigma)$ only swap postfix segments of runs. Examining Flip $(\sigma)$ is slightly more intricate, but it is not difficult to verify that the $\Sigma_{1}$ and $\Sigma_{2}$ which are swapped never contain any left to right minima values. Since the Swap map $\operatorname{Swap}_{a}$ is an involution, this gives the desired property.

132-peak-values. This is also treated via case-by-case analysis. 


\subsection{A bijection on permutations}

The recursions in Lemma 4.1 and Theorem 4.1 have the same structure, and thus allows us to construct an implicit bijection,

$$
\eta: S_{n} \rightarrow S_{n}
$$

with the main property that $\operatorname{PKV}(\sigma)=\operatorname{PKV}(\operatorname{runsort}(\eta(\sigma))$. The construction is recursive, and it is not canonical. For example, we shall impose the additional constraint that $\eta$ also preserves the set of descent bottoms, which is possible due to Lemma 4.7 .

We illustrate this idea with a concrete example.

Suppose $\eta(\sigma)=\sigma^{\prime}$ for $\sigma \in S_{n-1}$, where we know $\operatorname{PKV}(\sigma)=\operatorname{PKV}(\operatorname{runsort}(\eta(\sigma))$, and $\operatorname{DB}(\sigma)=\operatorname{DB}(\eta(\sigma))$, For example, with $\sigma=641325$ and $\sigma^{\prime}=645132$, suppose we have already determined that

$$
\eta(6,4,1,3,2,5)=(6,4,5,1,3,2) .
$$

By induction, we know that $\sigma$ and $\sigma^{\prime}$ have the same number of values of $a$ belonging to each respective case of Lemma 4.1 and Theorem 4.1, and they have the same set of descent bottoms, $(\{1,2,4\}$ in this case). In our example, we have the following situation:

\begin{tabular}{ccc} 
Case & $a$ for $\sigma$ & $a$ for $\sigma^{\prime}$ \\
\hline 1 & $\emptyset$ & $\emptyset$ \\
2 & 5 & 6 \\
3 & 3 & 3 \\
4 & 1 & 1 \\
$5 \mathrm{a}$ & 2 & 4 \\
$5 \mathrm{~b}$ & 4,6 & 2,5
\end{tabular}

For example, $a=3$ belongs to the second case for both $\sigma$ and $\sigma^{\prime}$. From the first five rows, we do not have any choice, and we set

$$
\begin{aligned}
& \eta(\mathcal{B}(\sigma, \emptyset)):=\mathcal{C}\left(\sigma^{\prime}, \emptyset\right) \\
& \eta(\mathcal{B}(\sigma, 5)):=\mathcal{C}\left(\sigma^{\prime}, 6\right) \\
& \eta(\mathcal{B}(\sigma, 3)):=\mathcal{C}\left(\sigma^{\prime}, 3\right) \\
& \eta(\mathcal{B}(\sigma, 1)):=\mathcal{C}\left(\sigma^{\prime}, 1\right) \\
& \eta(\mathcal{B}(\sigma, 2)):=\mathcal{C}\left(\sigma^{\prime}, 2\right)
\end{aligned}
$$

However, in the last row, any combination would ensure that $\eta$ behaves as desired on peaks and preserves the set of descent bottoms. For example, we can take

$$
\begin{aligned}
& \eta(\mathcal{B}(\sigma, 2)):=\mathcal{C}\left(\sigma^{\prime}, 4\right) \\
& \eta(\mathcal{B}(\sigma, 4)):=\mathcal{C}\left(\sigma^{\prime}, 2\right) \\
& \eta(\mathcal{B}(\sigma, 6)):=\mathcal{C}\left(\sigma^{\prime}, 6\right) .
\end{aligned}
$$

In conclusion, we have

$$
\begin{aligned}
& \eta(7,6,4,1,3,2,5)=(7,6,4,5,1,3,2) \\
& \eta(6,4,1,3,2,5,7)=(6,7,4,5,1,3,2) \\
& \eta(6,4,1,3,7,2,5)=(6,4,5,1,3,7,2) \\
& \eta(6,4,1,7,3,2,5)=(6,4,5,1,7,3,2) \\
& \eta(6,4,1,3,2,7,5)=(6,4,7,5,1,3,2) \\
& \eta(6,4,7,1,3,2,5)=(6,4,5,1,3,2,7) \\
& \eta(6,7,4,1,3,2,5)=(6,7,4,5,1,3,2),
\end{aligned}
$$

and thus, we have recursively extended the domain of $\eta$. We encourage the reader to verify that peak-values in the left hand side, are mapped to peak-values after runsort in the right hand side.

In general, there is no choice in Case 1 and Case 2, as there is always exactly one possible value of $a$ there. In Case 3 and Case 4, we have many possible combinations if we are only interested in preserving the number of peaks, but the choice is unique if we want to map PKV to SPV. In Case 3 for example, if $n$ is inserted after a peak-value $k$, then this value $k$ must be the same on both sides (but correspond to different values of $a$ ). But for Case $5 \mathrm{a}$ and Case 5b, there is some freedom, even if we demand that the set of descent bottoms is preserved. Incorporating additional statistics from Lemma 4.7 will not reduce this freedom further. Rather, it is necessary to find some combinatorial statistic that splits Case 5a and Case 5b further or makes the pairing canonical. 
Example 4.6. Here are the values of $\eta$, constructed with the above method. It turns out that this construction is canonical for the values presented below, but in general, choices have to be made.

\begin{tabular}{|c|c|c|c|c|c|c|c|}
\hline$\sigma$ & $\eta(\sigma)$ & $\sigma$ & $\eta(\sigma)$ & $\sigma$ & $\eta(\sigma)$ & $\sigma$ & $\eta(\sigma)$ \\
\hline 12 & 12 & 1234 & 1234 & 2314 & 2413 & 3412 & 3124 \\
\hline 21 & 21 & 1243 & 1243 & 2341 & 2134 & 3421 & 3241 \\
\hline 123 & 123 & 1324 & 1324 & 2413 & 2314 & 4123 & 4123 \\
\hline 132 & 132 & 1342 & 1342 & 2431 & 2143 & 4132 & 4132 \\
\hline 213 & 231 & 1423 & 1423 & 3124 & 3412 & 4213 & 4231 \\
\hline 231 & 213 & 1432 & 1432 & 3142 & 3142 & 4231 & 4213 \\
\hline 312 & 312 & 2134 & 2341 & 3214 & 3421 & 4312 & 4312 \\
\hline 321 & 321 & 2143 & 2431 & 3241 & 3214 & 4321 & 4321 \\
\hline
\end{tabular}

The discussion above (relying on Lemma 4.7) can be formulated as the following theorem which is the main result in this section.

Theorem 4.2. For $n \geq 1$, we have that

$$
\sum_{\pi \in S_{n}} \mathbf{x}_{\mathrm{DB}(\pi)} \mathbf{y}_{\mathrm{LRMin}(\pi)} \mathbf{z}_{\mathrm{PKV}}(\pi) \mathbf{w}_{\mathrm{PKV}(\pi)}=\sum_{\pi \in S_{n}} \mathbf{x}_{\mathrm{DB}(\pi)} \mathbf{y}_{\mathrm{LRMin}(\pi)} \mathbf{z}_{\mathrm{PKV}}(\pi) \mathbf{w}_{\mathrm{SPV}(\pi)} .
$$

Proof. Apply any of the bijections $\eta$, as constructed above, which preserves DB, LRMin, PKV*, and sends PKV to SPV.

We end this section with the following proposition, giving a new interpretation of http://oeis.org/ A202365.

Proposition 4.2. The numbers $B_{n}$ of permutations $\sigma$ such that $\operatorname{runsort}(\sigma)$ has a descent at position 2 satisfies the recurrence relation

$$
B_{n}=(n-1) !+(n-2) B_{n-1},
$$

with initial condition $B_{3}=2$. Consequently, $B_{n}=(n-2) !(n+1) \frac{n-2}{2}$ for $n \geq 3$. These also correspond to the counting sequence http://oeis. org/A202365 whose first few values are

$$
2,10,54,336,2400,19440,176400, \ldots
$$

Proof. Consider $\sigma \in S_{n-1}$. Inserting $n$ immediately after 1 in $\sigma$ and then run-sorting, gives a permutation $\operatorname{runsort}(\sigma) \in S_{n}$, whose first run is of the form $1 n$. Hence, we obtain $(n-1)$ ! permutations having a descent at position 2 .

We must now count the number of permutations $\sigma$, where we have a descent at position 2 , and $\sigma(2) \in$ $\{3,4, \ldots, n-1\}$. So now suppose $\sigma \in B_{n-1}$ which already has a descent at position 2 , and $\sigma(2)<n$. We can then insert $n$ anywhere, except after 1 or after $\sigma(2)$. The resulting permutation $\sigma^{\prime} \in S_{n}$ will then also have the property that runsort $\left(\sigma^{\prime}\right)$ lies in $B_{n}$. This method gives $(n-2) B_{n-1}$ additional permutations, and its straightforward to see that all elements in $B_{n}$ can be produced by exactly one of the two methods above.

\subsection{Probabilistic statements}

It is a standard exercise to compute the expected number of descents in a permutation.

Proposition 4.3. The expected number of descents in a permutation in $S_{n}$ is $(n-1) / 2$.

Proof. Let $I_{k}$ be the indicator variable that position $k \in[n-1]$ is a descent. We have that $\mathbb{E}\left[I_{k}\right]=1 / 2$, since the permutation with entries at $k$ and $k+1$ swapped are equally likely. Hence, with $\sigma \in S_{n}$,

$$
\mathbb{E}[\operatorname{des}(\sigma)]=\sum_{k=1}^{n-1} \mathbb{E}\left[I_{k}\right]=\frac{n-1}{2} .
$$

Given a uniformly random permutation in $S_{n}$, it is natural to ask what the expected number of descents is after performing runsort. We can now answer this in the following theorem.

Theorem 4.3 (See also $\left[29\right.$, p.110]). Let $\sigma \in S_{n}$, with $n \geq 2$ be uniformly chosen. Then

$$
\mathbb{E}[\operatorname{des}(\operatorname{runsort}(\sigma))]=\mathbb{E}[\operatorname{peaks}(\operatorname{runsort}(\sigma))]=\mathbb{E}[\operatorname{peaks}(\sigma)]=\frac{n-2}{3} .
$$


Proof. The first equality follows from the fact that the number of descents after run-sort is the same as the number of peaks, as we noted in Remark 2.1 The second identity follows immediately from Theorem 4.2, so it suffices to compute the expected number of peaks in a permutation. This has been done before in greater generality, see e.g., [10]. We include an argument below for the sake of completeness.

Recall from (17), the exponential generating function

$$
G(u, t):=\sum_{n \geq 0} \frac{u^{n}}{n !} \sum_{\pi \in S_{n}} t^{\operatorname{peaks}(\pi)}=\frac{\tan (u \sqrt{t-1})}{\sqrt{t-1}-\tan (u \sqrt{t-1})} .
$$

Note that for $\sigma \in S_{n}$, the expectation $\mathbb{E}[\operatorname{peaks}(\sigma)]$ is equal to the coefficient of $u^{n}$ in

$$
t \frac{\partial G(u, t)}{\partial t}=\sum_{n \geq 0} \frac{u^{n}}{n !} \sum_{\pi \in S_{n}} \operatorname{peaks}(\pi) \cdot t^{\text {peaks }(\pi)}
$$

evaluated at $t=1$. Differentiating $G(t, u)$ with respect to $t$ gives

$$
G_{t}(t, u)=\frac{t \sqrt{t-1} \sec ^{2}(u \sqrt{t-1})-\tan (u \sqrt{t-1})}{2 \sqrt{t-1}(\sqrt{t-1}-\tan (\sqrt{t-1}))^{2}} .
$$

Changing variables, with $\theta=u \sqrt{t-1}$, so that

$$
\sqrt{t-1}=\frac{\theta}{u} \text { and } 2 \sqrt{t-1}=\frac{2 \theta}{u} .
$$

Then (48) becomes

$$
\frac{u^{3}\left(\theta+\theta \tan ^{2}(\theta)-\tan \theta\right)}{2 \theta\left(\theta^{2}-2 \theta u \tan \theta+u^{2} \tan ^{2} \theta\right)} .
$$

We now require to find the limit of (48) as $t \rightarrow 1$. We note that in the change of variables, $\theta=u \sqrt{t-1}$, when $t=1$, then $\theta=0$. Now we obtain

$$
\lim _{\theta \rightarrow 0} \frac{u^{3}\left(\theta+\theta \tan ^{2}(\theta)-\tan \theta\right)}{2 \theta\left(\theta^{2}-2 \theta u \tan \theta+u^{2} \tan ^{2} \theta\right)},
$$

which is an indeterminate form $\frac{0}{0}$. Applying L'Hopitals rule three times, we obtain that (49) becomes

$$
\frac{4 u^{3}}{2\left(6-12 u+6 u^{2}\right)}=\frac{u^{3}}{3(u-1)^{2}}=\sum_{n=2}^{\infty} \frac{n-2}{3} u^{n} .
$$

Since $G(t, u)=\sum_{n \geq 0} B_{n}(t) \frac{u^{n}}{n !}$, we may now compare coefficients, and it follows immediately that

$$
\mathbb{E}[\operatorname{peaks}(\sigma)]=\frac{n-2}{3},
$$

for $\sigma \in S_{n}$ being uniformly chosen.

A result of a similar flavor was recently obtained by C. Defant [6], where the expected number of descents after stack-sorting is computed.

We end this section with a question.

Question 4.1. Let $\sigma \in S_{n}$ be a uniformly chosen permutation, and let $\sigma^{\prime}:=\operatorname{runsort}(\sigma)$. We can then consider the permutation matrix with entries equal to 1 at $\left(i, \sigma_{i}^{\prime}\right), i \in[n]$. Typically, this matrix (after scaling) looks like Figure 2, where a distinctive curve is seen. As $n \rightarrow \infty$ does this curve approach some limit curve?

\subsection{Possible peak-value sets}

In the recent article [8] the authors study for which sets $T \subseteq[n]$, there is at least one permutation with $T$ as peak-value set. Such a set is called admissible. They show [8, Thm. 1.8] that there are $\left(\begin{array}{c}n-1 \\ \left\lfloor\frac{n-1}{2}\right\rfloor\end{array}\right)$ such admissible sets.

Using our bijection, we see that a set $T$ is admissible if and only if it is the peak-value set of some run-sorted permutation. By now using the bijection sp-to-rsp and Proposition 2.1 we can see that $T \subseteq[n]$ is admissible if and only if there is some set partition $B_{1}\left|B_{2}\right| \cdots \mid B_{k}$ in $\mathcal{S P}(n-1)$ such that

$$
T=\left\{\max \left(B_{j}\right): j \in[k] \text { and }\left|B_{j}\right| \geq 2\right\} .
$$

From here, it should now be straightforward to count and characterize admissible sets. See also [7], where an alternative derivation of the number of admissible sets is provided. 


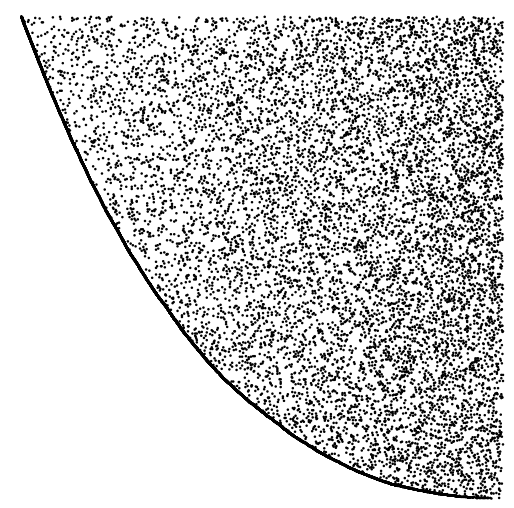

Figure 2: A random permutation matrix $\sigma^{\prime}$ after lexsort, for $n=20000$. The entries equal to 1 are shaded black.

\section{Run-sorted binary words}

Let $\operatorname{RSW}(a, b)$ denote the set of binary words of length $a+b$, with $a$ os and $b 1 \mathrm{~s}$, such that the runs in the word occur in lexicographical order. For example, 001010110111 is in $\operatorname{RSW}(5,7)$, since the runs 001, 01, 011 and 0111 are lexicographically ordered. Moreover, recall that the major index and the number of inversions of a permutation $\sigma \in S_{n}$ are defined as

$$
\operatorname{maj}(\sigma):=\sum_{j \in \operatorname{DES}(\sigma)} j, \quad \operatorname{inv}(\sigma):=\mid\{(i, j): 1 \leq i<j \leq n \text { and } \sigma(i)>\sigma(j)\} \mid .
$$

The first result in this section is the following identity (the proof is given later in Proposition 5.3).

Proposition 5.1. We have that

$$
\sum_{\substack{a \geq 0 \\ b \geq 0}}|\operatorname{RSW}(a, b)| q^{a} t^{b}=\prod_{i, j \geq 1} \frac{1}{1-q^{i} t^{j}} .
$$

Moreover, for any $a, b \geq 0, \operatorname{RSW}(a, b)$ is equal to the number of permutations $\sigma$ in $S_{n}$, such that $\operatorname{maj}(\sigma)=a$, $\operatorname{maj}\left(\sigma^{-1}\right)=b$.

Now, let the reverse-flip map revflip, acting on binary words $w$ of fixed length $n$, be defined as

$$
\operatorname{revflip}\left(w_{1}, \ldots, w_{n}\right):=\left(\hat{w}_{n}, \hat{w}_{n-1}, \ldots, \hat{w}_{2}, \hat{w}_{1}\right)
$$

where $\hat{w}_{j}:=1-w_{j}$. Finally, let $\Gamma(w):=\operatorname{runsort}(\operatorname{revflip}(w))$. We show below that $\Gamma: \operatorname{RSW}(a, b) \rightarrow \operatorname{RSW}(b, a)$ is a bijection. Moreover, we can also prove the following.

Proposition 5.2. There is a bijection between the set

$$
\{w \in \operatorname{RSW}(n, n): \Gamma(w)=w\}
$$

and $\mathcal{P}(n)$, the set of integer partitions of $n$. Moreover, for any $n \geq 1$,

$$
\sum_{\substack{w \in \operatorname{RSW}(n, n) \\ \operatorname{revflip}(w)=w}} x^{\beta(w)}=\sum_{\lambda \in \mathcal{P}(n)} x_{1}^{\lambda_{1}} x_{2}^{\lambda_{2}} \cdots x_{\ell}^{\lambda_{\ell}},
$$

where $x^{\beta(w)}=x_{1}^{\beta_{1}} \cdots x_{\ell}^{\beta_{\ell}}$, with $\beta_{j}$ denoting the number of 0 s in the $j^{\text {th }}$ run of $w$.

\subsection{From run-sorted binary words to bipartitions}

A bipartition (see [12]) is an array with two rows with positive integer entries,

$$
\left(\begin{array}{l}
\mathbf{u} \\
\mathbf{v}
\end{array}\right)=\left(\begin{array}{llll}
u_{1} & u_{2} & \cdots & u_{m} \\
v_{1} & v_{2} & \cdots & v_{m}
\end{array}\right),
$$

subject to the following conditions: 
- the entries in the top row are weakly increasing;

- if $u_{i}=u_{i+1}$ then $v_{i} \leq v_{i+1}$.

Let $\mathcal{B W}(a, b)$ be the set of bipartitions where the top row has total sum $a$, and the bottom row has total sum $b$. We note that bipartitions are a special case of biwords, where there are no conditions imposed on the array.

We shall now describe a bijection from $\operatorname{RSW}(a, b)$ to $\mathcal{B W}(a, b)$ as follows. Let $w \in \operatorname{RSW}(a, b)$ where $w=\rho_{1} \rho_{2} \cdots \rho_{r}$ are the runs of $w$. For each $i \in[r]$ we have a column we have a column $\left(\begin{array}{c}u_{i} \\ v_{i}\end{array}\right)$ in the bipartition, where $u_{i}$ is the number of $0 \mathrm{~s}$ in $\rho_{r+1-i}$ and $v_{i}$ is the number of $1 \mathrm{~s}$ in $\rho_{r+1-i}$.

We observe the following properties of this bijection.

- We have

$$
u_{1}+\cdots+u_{r}=a, \quad v_{1}+\cdots+v_{r}=b .
$$

- The top row is weakly increasing and if $u_{i}=u_{i+1}$ then $v_{i} \leq v_{i+1}$, since the word is run-sorted.

It is now straightforward to see that this map is indeed a bijection between $\operatorname{RSW}(a, b)$ and $\mathcal{B W}(a, b)$.

Example 5.1. Consider a binary word $w=00011011011101111 \in \mathrm{RSW}(6,11)$. The word $w$ has four runs, namely 00011,011,0111 and 01111. The bipartition corresponding to $w$ is given by

$$
\left(\begin{array}{llll}
1 & 1 & 1 & 3 \\
4 & 3 & 2 & 2
\end{array}\right)
$$

The following proposition is well-known, see e.g., [12]. It is one of the steps to show the classical Cauchyidentity via the Robinson-Schensted-Knuth correspondence, see [26, Ch.7].

Proposition 5.3. We have

$$
\sum_{a, b \geq 0}|\mathcal{B} \mathcal{W}(a, b)| q^{a} t^{b}=\prod_{i, j \geq 1} \frac{1}{1-q^{i} t^{j}} .
$$

Proof. By comparing coefficients, it is enough to show that $|\mathcal{B W}(a, b)|$ is the coefficient of $q^{a} t^{b}$ in the product

$$
\prod_{i, j \geq 1}\left(1+\left(q^{i} t^{j}\right)+\left(q^{i} t^{j}\right)^{2}+\left(q^{i} t^{j}\right)^{3}+\cdots\right)
$$

But now this is evident; the term $\left(q^{i} t^{j}\right)^{k}$ corresponds to the number of columns in the bipartition which are of the form $\left(\begin{array}{l}i \\ j\end{array}\right)$.

By using the bijection between bipartitions and run-sorted binary words, we can now, as promised, deduce Proposition 5.1.

We now introduce a set of permutations, which we shall see have the same cardinality as $\mathcal{B} \mathcal{W}(a, b)$. Let $\operatorname{MIP}(a, b) \subset S_{a+b}$ be the set of permutations $\sigma$ of $[a+b]$ with $\operatorname{maj}(\sigma)=a$, and $\operatorname{maj}\left(\sigma^{-1}\right)=b$. That is,

$$
\operatorname{MIP}(a, b):=\left\{\sigma \in S_{a+b}: \operatorname{maj}(\sigma)=a \text { and } \operatorname{maj}\left(\sigma^{-1}\right)=b\right\} .
$$

It is known, see e.g., [11, p.33] that for $n \geq 0$,

$$
\sum_{\sigma \in S_{n}} q^{\operatorname{maj}(\sigma)} t^{\operatorname{inv}(\sigma)}=\sum_{\sigma \in S_{n}} q^{\operatorname{maj}(\sigma)} t^{\operatorname{maj}\left(\sigma^{-1}\right)}
$$

so there are several combinatorial interpretations of the quantity $\operatorname{MIP}(a, b)$. See also the references in http: //oeis.org/A090806.

In [24], D. Roselle proved that

$$
\sum_{n \geq 0} \frac{z^{n}}{(q)_{n}(t)_{n}} \sum_{\pi \in S_{n}} t^{\operatorname{maj}(\pi)} q^{\operatorname{maj}\left(\pi^{-1}\right)}=\prod_{i, j \geq 0} \frac{1}{1-z q^{i} t^{j}},
$$

where $(q)_{n}:=(1-q)\left(1-q^{2}\right) \cdots\left(1-q^{n}\right)$.

A similar result was obtained by M. Cheema and T. Motzkin [5, Thm.4.1] and later generalized by A. Garsia and I. Gessel. In fact, it is possible to deduce the following proposition from Roselle's formula.

Proposition 5.4 (See [12, p. 299] and [5, Remark 6.2] ). We have

$$
\sum_{\substack{a \geq 0 \\ b \geq 0}}|\operatorname{MIP}(a, b)| q^{a} t^{b}=\prod_{i, j \geq 1} \frac{1}{1-q^{i} t^{j}}
$$


Combining (54) with Proposition 5.3, we see that

$$
\sum_{\substack{a \geq 0 \\ b \geq 0}}|\mathcal{B} \mathcal{W}(a, b)| q^{a} t^{b}=\sum_{\substack{a \geq 0 \\ b \geq 0}}|\operatorname{MIP}(a, b)| q^{a} t^{b}
$$

Problem 5.1. Find a bijection between the sets $\operatorname{RSW}(a, b)$ and $\operatorname{MIP}(a, b)$.

\subsection{Symmetric binary words}

Recall the bijection between run-sorted binary words and bipartitions. The following lemma is straightforward to prove.

Lemma 5.1. Suppose $w$ corresponds to the bipartition $\left(\begin{array}{l}\mathbf{u} \\ \mathbf{v}\end{array}\right)$. Then $\Gamma(w)$ has the bipartition $\left(\begin{array}{l}\mathbf{v}^{\prime} \\ \mathbf{u}^{\prime}\end{array}\right)$, which obtained from $\left(\begin{array}{l}\mathbf{u} \\ \mathbf{v}\end{array}\right)$ by first interchanging the two rows, and then sorting the columns so that the conditions of being a bipartition are met. Consequently, $\Gamma: \operatorname{RSW}(a, b) \rightarrow \operatorname{RSW}(b, a)$ is a bijection.

Example 5.2. We have $w=00011011011101111 \in \operatorname{RSW}(6,11)$, with bipartition

$$
\left(\begin{array}{llll}
1 & 1 & 1 & 3 \\
4 & 3 & 2 & 2
\end{array}\right)
$$

Then $\Gamma(00011011011101111)=00001000100100111$ and the latter word has the bipartition

$$
\left(\begin{array}{llll}
2 & 2 & 3 & 4 \\
3 & 1 & 1 & 1
\end{array}\right)
$$

Suppose now $\Gamma(w)=w$ for some $w \in \operatorname{RSW}(n, n)$. It follows from the bijection with bipartitions that the bipartition corresponding to $w$ is of the form

$$
\left(\begin{array}{cccc}
\lambda_{m} & \lambda_{m-1} & \cdots & \lambda_{1} \\
\lambda_{1} & \lambda_{2} & \cdots & \lambda_{m}
\end{array}\right)
$$

for some $\lambda \in \mathcal{P}(n)$, where $\lambda_{j}$ is the number of 0 s in the $j^{\text {th }}$ run of $w$. For example,

$$
001010101011 \leftrightarrow\left(\begin{array}{llll}
1 & 1 & 1 & 2 \\
2 & 1 & 1 & 1
\end{array}\right) \leftrightarrow(2,1,1,1) \in \mathcal{P}(5) .
$$

This reasoning then allows us to deduce Proposition 5.2.

\subsection{Descents in binary words after run-sorting}

Let $U_{k}(n)$ be defined as

$$
U_{k}(n):=\{w \in \mathcal{B} \mathcal{W}(n): \operatorname{des}(\operatorname{runsort}(w))=k\} .
$$

Proposition 5.5. We have that for any $n \geq 1, U_{0}$ is the set of binary words of length $n$ matching the regular expression $1 * 0 * 1 * 0 *$. Such words can be split into two cases, matching either $1 * 0 *$ or $1 *(0+1+) 0 *$. These are the words of the form

$$
\begin{cases}\underbrace{1 \ldots 1}_{a \geq 0} \underbrace{0 \ldots 0}_{d \geq 0} & a+d=n, \text { or } \\ \underbrace{1 \ldots 1}_{a \geq 0} \underbrace{0 \ldots 0}_{b \geq 1} \underbrace{1 \ldots 1}_{c \geq 1} \underbrace{0 \ldots 0}_{d \geq 0} & a+b+c+d=n .\end{cases}
$$

Moreover, for $k \geq 2$, the set $U_{k-1}(n)$ consists of all binary words of length $n$ matching the regular expression $1 *(0+1+)\{\mathrm{k}\} 0 *$, i.e., words of the form

$$
\underbrace{1 \ldots 1}_{a \geq 0} \underbrace{0 \ldots 0}_{b_{1} \geq 1} \underbrace{1 \ldots 1}_{c_{1} \geq 1} \underbrace{0 \ldots 0}_{b_{2} \geq 1} \underbrace{1 \ldots 1}_{c_{2} \geq 1} \cdots \underbrace{0 \ldots 0}_{b_{k} \geq 1} \underbrace{1 \ldots 1}_{c_{k} \geq 1} \underbrace{0 \ldots 0}_{d \geq 0},
$$

where $a+\left(b_{1}+c_{1}+b_{2}+c_{2}+\cdots+b_{k}+c_{k}\right)+d=n$.

Proof. First, note that every word in $\mathcal{B W}(n)$ belongs to exactly one of the sets $U_{k}(n)$. The binary words with 0 or 1 subwords equal to 01 , are captured in (55), while binary words with $k$ subwords of the form 01 belong to $(56)$.

Hence, it suffices to show that the words in $U_{k}(n)$ have exactly $k$ descents after run-sort, which is straightforward.

\footnotetext{
TSee https://en.wikipedia.org/wiki/Regular\textunderscore_expression.
} 
Analogous to the results in Section 4, we can study the distribution of descents in binary words after run-sorting.

Theorem 5.1. Let $C_{n}(t):=\sum_{w \in \mathcal{B} \mathcal{W}(n)} t^{\operatorname{des}(\operatorname{runsort}(w))}$. Then

$$
C_{n}(t)=\left(\begin{array}{c}
n+1 \\
3
\end{array}\right)+(n+1)+\sum_{k=2}^{n} t^{k-1}\left(\left(\begin{array}{c}
n \\
2 k
\end{array}\right)+\left(\begin{array}{c}
n \\
2 k+1
\end{array}\right)\right) .
$$

In particular, the number of binary words of length $n$ with no descents after run-sort, is given by http: // oeis. org/A000125, the Cake numbers, $\left(\begin{array}{c}n+1 \\ 3\end{array}\right)+(n+1)$.

Proof. The case $k=0$ is straightforward; there are exactly $n+1$ words of the form $1 * 0 *$. That the words in the second case of $(55)$ are $\left(\begin{array}{c}n+1 \\ 3\end{array}\right)$ can be proved in the same manner as the $k \geq 2$ situation, that is, that the number of words of length $n$ of the form in (56), is the sum $\left(\begin{array}{c}n \\ 2 k\end{array}\right)+\left(\begin{array}{c}n \\ 2 k+1\end{array}\right)$.

Observe that for each binary word of the form in (56), we have segments of identical digits of lengths $a \geq 0$, $d \geq 0, b_{i} \geq 1$ and $c_{i} \geq 1$, for $1 \leq i \leq k$. For each such segment of identical digits, we need to choose the position of the last digit. When $a=0$, there are $k$ segments of 0 s and $k$ segments of 1 s, hence giving us a total of $2 k$ positions of the last 0 and 1 in each of these segments. The positions of these digits are chosen among the $n$ positions. Hence we have a total of $\left(\begin{array}{c}n \\ 2 k\end{array}\right)$ words of the form in (56) with $a=0$.

Moreover, when $a \geq 1$, then the first segment contains at least one digit. Hence we need to choose the position of the last digit for this segment also, on top of the $2 k$ positions from segments of lengths $b_{i}$ and $c_{i}$. Thus we have $2 k+1$ positions to be chosen out of $n$ total positions. This gives $\left(\begin{array}{c}n \\ 2 k+1\end{array}\right)$ binary words of the form in (56) with $a>0$, and we are done.

\subsection{Expected number of descents in a run-sorted binary word}

From the formula in Equation (57) we can easily produce the following exponential generating function:

$$
\begin{aligned}
H(u, t) & :=\sum_{n \geq 0} \frac{u^{n}}{n !}\left(\left(\begin{array}{c}
n+1 \\
3
\end{array}\right)+(n+1)+\sum_{k=2}^{n} t^{k-1}\left(\left(\begin{array}{c}
n \\
2 k
\end{array}\right)+\left(\begin{array}{c}
n \\
2 k+1
\end{array}\right)\right)\right) \\
& =\frac{e^{u}(\sinh (\sqrt{t} u)+\sqrt{t}((t-1)(u+1)+\cosh (\sqrt{t} u)))}{t^{3 / 2}} .
\end{aligned}
$$

Mathematica produces the generating function in (58) automatically by using the command

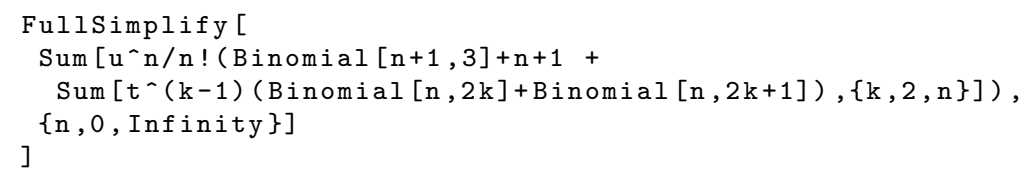

From $H(u, t)$, we can compute the expected number of descents in a binary word after run-sort. It is given by first taking the $t$-derivative of $H(u, t)$, then substituting $t=1$. Finally, we extract the coefficient of $u^{n}$, multiply by $n$ !, and divide by $2^{n}$ (the total number of binary words). That is,

$$
\mathbb{E}[\operatorname{des}(\operatorname{runsort}(w))]=\frac{n !}{2^{n}}\left[u^{n}\right] H_{t}^{\prime}(u, 1) .
$$

Mathematica can again do all these steps for us. If we let genFunc denote the exponential generating function $H(u, t)$, then the command

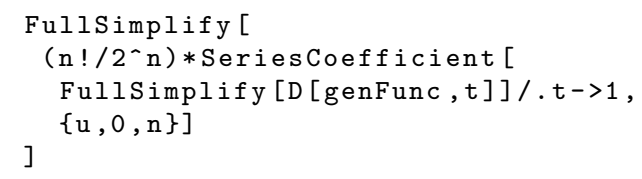

gives us $\frac{n-5}{4}+2^{-n}(n+1)$.

\section{Acknowledgement}

The second author acknowledges the financial support extended by the Swedish Sida Phase-IV bilateral program with Makerere University. Special thanks go to Jörgen Backelin and Paul Vaderlind for all their valuable inputs and suggestions. We are also thankful for suggestions from Petter Brändén, in particular the proof of Theorem 3.5. We thank M. Rubey for suggesting to look at left-to-right minima in Lemma 4.7. Many thanks to colleagues from CoRS - Combinatorial Research Studio for lively discussions. 


\section{References}

[1] P. Brändén, J. Haglund, M. Visontai, and D.G. Wagner, Proof of the monotone column permanent conjecture, In Notions of Positivity and the Geometry of Polynomials (pp. 63-78), Springer, Basel, 2011.

[2] P. Brändén and M. Leander, Multivariate p-Eulerian polynomials, arXiv:1604.04140.

[3] P. Brändén, Unimodality, log-concavity, realrootedness and beyond, In Handbook of Enumerative Combinatorics 87 (2015), 437-483.

[4] D. Callan, Pattern avoidance in "flattened" partitions, Discrete Math. 309 (2009), 4187-4191.

[5] M.S. Cheema and T.S. Motzkin, Multipartitions and multipermutations, Proc. Symp. Pure Math, 19 (1971), $37-39$.

[6] C. Defant, Troupes, cumulants, and stack-sorting, arXiv:2004.11367.

[7] R. Domagalski, J. Liang, Q. Minnich, B.E. Sagan, J. Schmidt, and A. Sietsema, Pinnacle Set Properties, arXiv:2105.10388.

[8] R. Davis, S.A. Nelson, T.K. Petersen, and B.E. Tenner, The pinnacle set of a permutation, Discrete Math. 341 (2018), 3249-3270.

[9] R. Ehrenborg and E. Steingrimsson, The excedance set of a permutation, Adv. in Appl. Math. 24 (2000), 284-299.

[10] J. Fulman, G.B. Kim, and S. Lee, Central limit theorem for peaks of a random permutation in a fixed conjugacy class of $S_{n}$, arXiv:1902.00978.

[11] D. Foata, Distributions Eulériennes et Mahoniennes sur le groupe des permutations, Higher Combinatorics (pp. 27-49), Springer, 1977.

[12] A.M. Garsia and I. Gessel, Permutation statistics and partitions, Adv. Math. 31 (1979), 288-305.

[13] J. Haglund and M. Visontai, Stable multivariate Eulerian polynomials and generalized Stirling permutations, European J. Combin. 33 (2012), 477-487.

[14] S. Kitaev, Introduction to partially ordered patterns, Discrete Appl. Math. 155 (2007), 929-944.

[15] J. Baptiste and J. Liagre, Sur la probabilit de l'existence d'une cause d'erreur rgulire dans unesrie d'observations, Bull. Acad. r. Belg 22 (1855), 7-55.

[16] J.D. Leake and N.R. Ryder, Generalizations of the matching polynomial to the multivariate independent polynomial, Algebr. Comb. 2 (2019), 781-802.

[17] T.Y.H. Liu and A.Q. Zhang, On pattern avoiding flattened set partitions, Acta Math. Sin. (Engl. Ser.) 31 (2015), 1923-1928.

[18] P.A. MacMahon, Combinatorial Analysis, Volume I \& II, Chelsea Publishing Co., New York, 1960.

[19] R. Mantaci and F. Rakotondrajao, Exceedingly deranging!, Adv. in Appl. Math. 30 (2003), 177-188.

[20] T. Mansour and M. Shattuck, Pattern avoidance in flattened permutations, Pure Math. Appl. (Pu.M.A.) 22 (2011), 75-86.

[21] T. Mansour, M. Shattuck, and S. Wagner, Counting subwords in flattened partitions of sets, Discrete Math. 338 (2015), 1989-2005.

[22] O. Nabawanda and F. Rakotondrajao, Sets of Flattened Partitions with Forbidden Patterns, J. Integer Seq. 24 (2021), Article 21.1.5.

[23] O. Nabawanda, F. Rakotondrajao, and A.S. Bamunoba, Run Distribution over Flattened Partitions, J. Integer Seq. 23 (2020), Article 20.9.6.

[24] D. P. Roselle, Coefficients associated with the expansion of certain products, Proc. Amer. Math. Soc. 45 (1974), 144-150.

[25] E. S. W. Shiu, Steffensen's poweroids, Scand. Actuar. J. 1982 (1982), 123-128.

[26] R. P. Stanley, Enumerative Combinatorics, Volume II, Cambridge University Press, Cambridge, UK, 2001.

[27] J. F. Steffensen, The poweroid, an extension of the mathematical notion of power, Acta Math. 73 (1941), 333-366.

[28] D. G. Wagner, Total positivity of Hadamard products, J. Math. Anal. Appl. 163 (1992), 459-483.

[29] D. Warren and E. Seneta, Peaks and Eulerian numbers in a random sequence, J. Appl. Probab. 33 (1996), 101-114. 